\title{
On finite linear systems containing strict inequalities
}

\author{
M.M.L. Rodríguez* J. Vicente-Pérez ${ }^{\dagger}$ \\ August 29, 2016 - Submitted version
}

\begin{abstract}
This paper deals with linear systems containing finitely many weak and/or strict inequalities whose solution sets are referred to as evenly convex polyhedral sets. The classical Motzkin Theorem states that every (closed convex) polyhedron is the Minkowski sum of a convex hull of finitely many points and a finitely generated cone. In this sense, similar representations for evenly convex polyhedra have been recently given by using the standard version for classical polyhedra. In this work, we provide a new dual tool that completely characterizes finite linear systems containing strict inequalities and it constitutes the key for obtaining a generalization of Motzkin Theorem for evenly convex polyhedra.
\end{abstract}

Keywords: linear systems; strict inequalities; polyhedra; even convexity; duality.

\section{Introduction}

The solution sets of linear systems containing finitely many weak inequalities, the socalled polyhedra, are one of the cornerstones of linear and quadratic programming and because of this, they have been extensively studied last century (see, for instance, $\lfloor 14,15,17,22,24\rfloor$ and references therein). Furthermore, polyhedra enjoy important applications in formal methods $[2,3\rfloor$, combinatorial optimization $[23\rfloor$, decision making theory [20], and in other areas where linear programming play a central role. Thus, the celebrated Farkas lemma [6], the most well-known theorem of the alternative which characterizes the consequence relations of a finite homogeneous system by means of the convex cone generated by the coefficient vectors of the system, is the fundamental principle of the theory of linear inequality systems. The later extension of Farkas lemma to nonhomogeneous linear systems is called Farkas-Minkowski theorem (see $[25\rfloor$ ).

Regarding solvability of finite linear inequality systems, Fourier proposed in 1897 a method (known as the Fourier-Motzkin elimination method) consisting of successive

\footnotetext{
*Department of Mathematics, University of Alicante, 03071 Alicante, Spain. Email address: marga.rodriguez@ua.es

${ }^{\dagger}$ Department of Economics, University of Alicante, 03071 Alicante, Spain. Email address: jose.vicente@ua.es
} 
elimination of variables. A complete description of this method can be found in [16] where systems are assumed to contain both weak and strict inequalities. A dual form of the Fourier-Motzkin elimination method was used in [18] to show that every polyhedral cone (the solution set of a finite homogeneous linear inequality system) is finitely generated, that is, its elements are the nonnegative linear combinations of a finite set of vectors. On the other hand, with the simpler version of that elimination method for systems containing just weak inequalities, in [27] was proved that every finitely generated cone is polyhedral. By using the same technique, it is also shown that every polytope (the convex hull of a finite set of points) and every Minkowski sum of a polytope and a finitely generated cone are also polyhedra.

Motzkin [19] proved that any polyhedron can be expressed as the Minkowski sum of a polytope and a polyhedral cone. This result together with Minkowski and Weyl theorems provide the characterization of polyhedra as those sets which can be expressed as the sum of a polytope and a finitely generated cone. Thus, a polyhedron can be described by using either a finite linear system of weak inequalities (external representation) or by using a system of generating points and rays (internal representation) in which any point of the polyhedron is obtained as the sum of a convex combination of the generating points and a nonnegative combination of the generating rays. These two ways of representing a polyhedron are dual in the sense that each representation can be computed starting from the other one, and the conversion procedure is called the double description method. That double representation is very useful in order to establish the main basic operations on the family of polyhedra. Thus, to obtain the intersection of two polyhedron, the best representation is the external one while internal representations are more suitable for computing the sum of two polyhedra.

Zhu $[30\rfloor$ provided a characterization of the consistency for a linear system containing an arbitrary number (possibly infinite) of weak inequalities in terms of the so-called characteristic cone. In the particular case of finite systems, the characteristic cone is a finitely generated cone depending on the coefficient vectors of the system and it can be considered as a dual representation for the solution set of that system. Moreover, this cone allowed to reformulate Farkas-Minkowski theorem, to simplify the procedure for obtaining an internal representation of a polyhedron from an external one (see $[9\rfloor$ ), and to characterize the set containment of polyhedra (see [8]).

In this work we deal with finite linear systems containing strict inequalities. These kind of systems were already considered by Fourier and Motzkin in their elimination method. Some results, presented as alternative theorems, were obtained in the late 19th and early 20th centuries. Regarding their solution sets, [7」 introduced the concept of evenly convex set to refer to a set which is the intersection of a family (possibly infinite) of open halfspaces and, since any closed halfspace is an intersection of infinitely many open halfspaces, evenly convex sets can be considered as the solution sets of linear systems containing an arbitrary number of either weak or strict inequalities (see $[10,12])$. Inspired by this definition, we will say that a set is an evenly convex polyhedral set (e-polyhedron, in brief) if it is the intersection of finitely many closed and/or open halfspaces. Clearly, the family of e-polyhedra contains the family of (closed convex) polyhedra, and it is contained in the family of evenly convex sets.

The notion of e-polyhedra has appeared in the literature with other names. Thus, 
this family was introduced as wholefaced polyhedra in [26], copolyhedra in [13], not necessarily closed (NNC, in brief) convex polyhedra in [1], G-polyhedra in [29] and semiclosed polyhedra in [28]. In all those papers, e-polyhedra were defined by means of their external representations, that is, as the solution sets of finite linear systems possibly containing strict inequalities. The family of e-polyhedra play an important role, for instance, in the study of piecewise linear programs: $[28\rfloor$ proved that the Pareto solution set of a piecewise linear multiobjective optimization problem is the union of finitely many e-polyhedra. By exploiting the fact that an e-polyhedron is obtained by removing some faces from a polyhedron, [5] obtained an internal representation for e-polyhedra which was applied to show that the image of an e-polyhedron under a continuous function is always an e-polyhedron, and to study sensitivity in a piecewise linear program with possible discontinuity $\lfloor 4\rfloor$.

A different internal representation for e-polyhedra was recently given by $[1]$ related to the field of automatic analysis and verification of hardware and software systems (see $[2]$ for a survey of applications of polyhedral computations in this area). In particular, $[3\rfloor$ began to develop a library of polyhedral algorithms for the manipulation of both polyhedra and e-polyhedra using the double description method, the so-called Parma Polyhedra Library. The significance of having an internal representation relies on the fact that the ability to switch from one to another representation can be usefully exploited to provide simple implementations for the basic operations on e-polyhedra. The internal representation for e-polyhedra provided in [1] was obtained by using an indirect proof based on the standard version of Motzkin theorem. Thus, both representations for e-polyhedra obtained in $\lfloor 1\rfloor$ and $\lfloor 5\rfloor$ were shown by using the well-know internal representation for the subfamily of (closed convex) polyhedra.

Our key contributions are outlined as follows. We first introduce in Section 3 a new dual cone, called extended characteristic cone, for analyzing finite linear systems containing strict inequalities. By using this cone, we shall obtain extensions of Zhu and Farkas-Minkowski theorems. Moreover, we recover some well-known Farkas-type results and we study the role of this cone in the containment problem of e-polyhedra. In Section 4, we use the properties of the extended characteristic cone in order to prove extended versions of Weyl and Motzkin theorems recovering the internal representation for e-polyhedra given in [1]. Thus, as every polyhedron is an e-polyhedron, the classical representation for polyhedra follows as a particular case. The given proofs provide a direct method to obtain an internal representation of an e-polyhedron from an external one, and vice versa. We conclude the paper by showing a minimal internal representation of an e-polyhedron.

\section{Preliminaries}

We start by introducing the necessary notation and basic results to be used later. Throughout the paper, the standard inner product of two vectors $u, v \in \mathbb{R}^{n}$ will be denoted by $\langle u, v\rangle$. We also denote by $0_{n}, \mathbb{R}_{+}^{n}$ and $\mathbb{R}_{++}^{n}$, the zero vector, the nonnegative orthant and the positive orthant in $\mathbb{R}^{n}$, respectively. Given a nonempty set $X \subset \mathbb{R}^{n}$, we denote by $\operatorname{span} X, \operatorname{cl} X, \operatorname{rint} X, \operatorname{conv} X$ and cone $X$ the linear subspace of $\mathbb{R}^{n}$ 
spanned by $X$, the closure of $X$, the relative interior of $X$, the convex hull of $X$ and the convex cone generated by $X$ and the origin, respectively. Moreover, we define $\operatorname{span} \emptyset=\operatorname{cone} \emptyset=\left\{0_{n}\right\}$. If $X$ is a nonempty finite set, then it is said that conv $X$ is a polytope and cone $X$ is a finitely generated cone. Recall that a polyhedron is the solution set of a linear inequality system containing a finite number of weak inequalities, and so every polyhedron is a closed convex set. Thus, a set is a polytope if and only if it is a bounded polyhedron. Polyhedra that are also cones are referred to as polyhedral cones and they are intersections of finite families of closed halfspaces containing the origin in their boundaries. The following result is the classical Minkowski-Weyl Theorem.

Lemma 2.1 A cone is polyhedral if and only if it is finitely generated.

The Minkowski sum of $X, Y \subset \mathbb{R}^{n}$ is the set $X+Y:=\{x+y: x \in X, y \in Y\}$, whereas the recession cone of a nonempty convex set $X \subset \mathbb{R}^{n}$ is

$$
\operatorname{rec}(X):=\left\{d \in \mathbb{R}^{n}: x+t d \in X \text { for all } t \geq 0 \text { and for all } x \in X\right\}
$$

The (negative) polar cone of a nonempty convex cone $X \subset \mathbb{R}^{n}$ is defined to be the set

$$
X^{\circ}:=\left\{v \in \mathbb{R}^{n}:\langle v, x\rangle \leq 0 \text { for all } x \in X\right\} .
$$

Namely, if $X$ is a nonempty finitely generated cone, say $X=$ cone $\left\{d_{1}, \ldots, d_{m}\right\}$, then $X^{\circ}=\left\{v \in \mathbb{R}^{n}:\left\langle v, d_{i}\right\rangle \leq 0\right.$ for all $\left.i=1, \ldots, m\right\}$, which is a polyhedral cone. Moreover, as a consequence of the Farkas Lemma for cones, one has $X^{\circ \circ}=X$.

Given a nonempty convex set $X \subset \mathbb{R}^{n}$, a subset $C \subset X$ is said to be an exposed face of $X$ if there exists $a \in \mathbb{R}^{n}$ such that $C=\arg \max \{\langle a, x\rangle: x \in X\}$. Thus, the exposed faces of $X$ (except $X$ itself and possibly the empty set) are the sets of the form $X \cap H$, where $H$ is a nontrivial supporting hyperplane to $X$. If $X \subset \mathbb{R}^{n}$ is a convex set such that its closure is a polyhedron, then all the faces of $X$ are exposed. A face $C$ of a convex set $X$ is minimal if there is no nonempty face $D$ of $X$ such that $D \subsetneq C$.

According to [7], a set is said to be evenly convex if it is the intersection of a family (possibly infinite) of open halfspaces. Since any closed halfspace is the intersection of infinitely many open halfspaces, a set is evenly convex if and only if it is the solution set of a certain linear system containing an arbitrary number (possibly infinite) of both weak and strict inequalities. From the geometric point of view, a nonempty convex set $X \subset \mathbb{R}^{n}$ is evenly convex if and only if for every $\bar{x} \in \mathbb{R}^{n} \backslash X$ there exists a hyperplane $H$ such that $\bar{x} \in H$ and $H \cap X=\emptyset$. Moreover, evenly convex sets can also be characterized as those sets which are the result of eliminating from their closures the union of a certain family of exposed faces. See $[10]$ and $[12]$ for a comprehensive review on evenly convex sets. On the other hand, a convex set $X$ is said to be wholefaced [19] if for any $x \in X$ and $y \in \operatorname{cl} X$ one has $[x, y[\subset X$. It is well-known (cf. [7]) that every evenly convex set is wholefaced.

Dual cones for characterizing closed convex and evenly convex sets have been widely studied in [8]. Thus, the weak dual cone of $X \subset \mathbb{R}^{n}$ is defined to be $K \leq:=\{(a, b) \in$ $\left.\mathbb{R}^{n} \times \mathbb{R}:\langle a, x\rangle \leq b, \forall x \in X\right\}$. Hence, if $X$ is a nonempty closed convex set, the standard hyperplane separation arguments yields $X=\left\{x \in \mathbb{R}^{n}:\langle a, x\rangle \leq b, \forall(a, b) \in K^{\leq}\right\}$. 
Moreover, if $Y \subset \mathbb{R}^{n}$ is another nonempty closed convex set with associated weak dual cone $M \leq$, one has $X \subset Y$ if and only if $M \leq \subset K^{\leq}$. Analogously, the strict dual cone of $X \subset \mathbb{R}^{n}$ is defined to be $K^{<}:=\left\{(a, b) \in \mathbb{R}^{n} \times \mathbb{R}:\langle a, x\rangle<b, \forall x \in X\right\}$. Thus, if $X$ is a nonempty evenly convex set, its symmetric expression is now a direct consequence of the characterization of the evenly convex sets by means of the strong separation property from external points and so $X=\left\{x \in \mathbb{R}^{n}:\langle a, x\rangle<b, \forall(a, b) \in K^{<}\right\}$. Moreover, if $Y \subset \mathbb{R}^{n}$ is another nonempty evenly convex set with associated strict dual cone $M^{<}$, one has $X \subset Y$ if and only if $M^{<} \subset K^{<}$.

\section{Duality for finite linear inequality systems}

We begin this section by introducing the notion of evenly convex polyhedral set which is inspired by the definition of evenly convex set. As summarized in Section 1, this notion has been differently referred to in the literature.

Definition 3.1 An evenly convex polyhedral set (e-polyhedron, in brief) in $\mathbb{R}^{n}$ is the solution set of a linear inequality system containing a finite number of either weak or strict inequalities. We shall denote by $P$ the solution set of

$$
\sigma:=\left\{\left\langle a_{t}, x\right\rangle \leq b_{t}, t \in W ;\left\langle a_{t}, x\right\rangle<b_{t}, t \in S\right\}
$$

where $\left(a_{t}, b_{t}\right) \in \mathbb{R}^{n} \times \mathbb{R}$ for all $t \in T:=S \cup W, S \cap W=\emptyset$ and $|T|<\infty$.

Clearly, every (closed convex) polyhedron (whenever $S=\emptyset$ ) is an e-polyhedron, and every open convex polyhedron (whenever $W=\emptyset$ ) is also an e-polyhedron. Furthermore, every e-polyhedron is obviously an evenly convex set. We shall denote by $\bar{P}$ the polyhedron which is the solution set of $\bar{\sigma}$, the relaxed system associated to $\sigma$,

$$
\bar{\sigma}:=\left\{\left\langle a_{t}, x\right\rangle \leq b_{t}, t \in T\right\}
$$

According to $[10$, Proposition 1.1], if $P \neq \emptyset$ then $\mathrm{cl} P=\bar{P}$. The so-called characteristic cone associated to $\bar{\sigma}$ is defined to be the finitely generated cone

$$
K:=\operatorname{cone}\left\{\left(a_{t}, b_{t}\right), t \in T ;\left(0_{n}, 1\right)\right\},
$$

which indeed coincides with the weak dual cone of $\bar{P}$. The polar cone of $K$ is denoted by $F_{n+1}$ and it is given by

$$
F_{n+1}=\left\{(x, y) \in \mathbb{R}^{n} \times \mathbb{R}: \begin{array}{rl}
\left\langle a_{t}, x\right\rangle+b_{t} y & \leq 0, t \in T \\
y & \leq 0
\end{array}\right\} .
$$

According to $[10$, Proposition 3.3], one has that if $P \neq \emptyset$, then

$$
\operatorname{rec}(P)=\operatorname{rec}(\bar{P})=\left\{d \in \mathbb{R}^{n}:\left\langle a_{t}, d\right\rangle \leq 0, t \in T\right\}
$$

which is a polyhedral cone. As a consequence of Lemma 2.1, it is a finitely generated cone and so, $\operatorname{rec}(P)=$ cone $\left\{d_{1}, \ldots, d_{r}\right\}$ for some extreme rays $d_{1}, \ldots, d_{r} \in \mathbb{R}^{n}$. Moreover, if $P \neq \emptyset$, then $P$ is bounded if and only if $\operatorname{rec}(P)=\left\{0_{n}\right\}$.

Next we introduce a new dual cone for analyzing finite linear inequality systems. 
Definition 3.2 The extended characteristic cone associated to the system $\sigma$ in (1) is the finitely generated cone

$$
\mathcal{K}:=\text { cone }\left\{\left(a_{t}, b_{t}, 0\right), t \in W ;\left(a_{t}, b_{t},-1\right), t \in S ;\left(0_{n}, 1,-1\right) ;\left(0_{n}, 0,1\right)\right\} .
$$

The polar cone of $\mathcal{K}$ is denoted by $F_{n+2}$ and it is given by

$$
F_{n+2}=\left\{(x, y, z) \in \mathbb{R}^{n} \times \mathbb{R} \times \mathbb{R}: \begin{array}{rl}
\left\langle a_{t}, x\right\rangle+b_{t} y & \leq 0, t \in W \\
\left\langle a_{t}, x\right\rangle+b_{t} y-z & \leq 0, t \in S, \\
y-z & \leq 0 \\
z & \leq 0
\end{array}\right\} .
$$

Next result establishes some relations between the extended characteristic cone of a finite linear inequality system and the characteristic cone of its associated relaxed system. For that purpose, we shall denote by $\pi$ the projection into $\mathbb{R}^{n+1}$ defined by $\pi(x, y, z):=(x, y)$ for every $(x, y, z) \in \mathbb{R}^{n} \times \mathbb{R} \times \mathbb{R}$.

Lemma 3.3 The following statements hold:

(i) $K \times \mathbb{R}_{+} \subsetneq \mathcal{K}$.

(ii) $\mathcal{K} \subset K \times \mathbb{R}$.

(iii) $K=\pi(\mathcal{K})$.

(iv) $F_{n+1}=\pi\left(F_{n+2}\right)$.

Proof. $(i)$ Let $(a, b, c) \in K \times \mathbb{R}_{+}$. As $(a, b) \in K$, there exist $\lambda_{t} \geq 0$ for all $t \in W \cup S$ and $\alpha \geq 0$ such that

$$
(a, b)=\sum_{t \in W \cup S} \lambda_{t}\left(a_{t}, b_{t}\right)+\alpha\left(0_{n}, 1\right) .
$$

By letting $\beta:=c+\sum_{t \in S} \lambda_{t}+\alpha \geq 0$, one has

$$
(a, b, c)=\sum_{t \in W} \lambda_{t}\left(a_{t}, b_{t}, 0\right)+\sum_{t \in S} \lambda_{t}\left(a_{t}, b_{t},-1\right)+\alpha\left(0_{n}, 1,-1\right)+\beta\left(0_{n}, 0,1\right)
$$

and so $(a, b, c) \in \mathcal{K}$. Moreover, the inclusion is always strict since $\left(0_{n}, 1,-1\right) \in \mathcal{K}$ but $\left(0_{n}, 1,-1\right) \notin K \times \mathbb{R}_{+}$.

Statement ( $i i$ ) easily follows from the definitions of $K$ and $\mathcal{K}$.

(iii) Let $(a, b) \in K$. Statement $(i)$ yields $(a, b, c) \in \mathcal{K}$ for all $c \geq 0$, and so $(a, b)=\pi(a, b, c)$. Thus, $K \subset \pi(\mathcal{K})$. The reverse inclusion easily follows from $(i i)$.

(iv) If $(x, y) \in F_{n+1}$, then $(x, y, 0) \in F_{n+2}$. Conversely, if $(x, y, z) \in F_{n+2}$, one has $\left\langle a_{t}, x\right\rangle+b_{t} y \leq 0$ for all $t \in W,\left\langle a_{t}, x\right\rangle+b_{t} y \leq\left\langle a_{t}, x\right\rangle+b_{t} y-z \leq 0$ for all $t \in S$ (as $z \leq 0)$ and $y \leq z \leq 0$, and so $(x, y) \in F_{n+1}$.

A straightforward consequence of the above lemma is that $(a, b, 0) \in \mathcal{K}$ if and only if $(a, b) \in K$, which collapses to $\pi(\mathcal{K})=\pi\left(\mathcal{K} \cap\left(\mathbb{R}^{n+1} \times\{0\}\right)\right)$. The inclusion in (ii) can be strict as we can see in the following example: 
Example 3.4 Let consider the system $\sigma=\left\{-x_{1}-x_{2} \leq-2,-x_{1}+x_{2}<0\right\}$ in $\mathbb{R}^{2}$. By solving the linear system

$$
(-2,-1,-3,-1)=\alpha(-1,-1,-2,0)+\beta(-1,1,0,-1)+\gamma(0,0,1,-1)+\mu(0,0,0,1),
$$

one has $\alpha=\frac{3}{2}, \beta=\frac{1}{2}, \gamma=0$ and $\mu=\frac{-1}{2}<0$. This shows that $(-2,-1,-3,-1) \notin \mathcal{K}$. However, one has $(-2,-1,-3) \in K$ and so $(-2,-1,-3,-1) \in K \times \mathbb{R}$.

\subsection{Existence of solutions}

This section is devoted to characterize the consistency of finite linear systems containing strict inequalities.

Lemma 3.5 Let $P \subset \mathbb{R}^{n}$ be the solution set of the system $\sigma$ in (1). The following statements hold:

(i) If $P \neq \emptyset$, then $\bar{x} \in P$ if and only if $(\bar{x},-1, \varepsilon) \in F_{n+2}$ for some $\varepsilon \in[-1,0[$.

(ii) $P=\emptyset$ if and only if $z=0$ for all $(x, y, z) \in F_{n+2}$.

Proof. (i) Assume that $P \neq \emptyset$ and let $\bar{x} \in P$. This means that $\left\langle a_{t}, \bar{x}\right\rangle-b_{t} \leq 0$ for all $t \in W$ and $\left\langle a_{t}, \bar{x}\right\rangle-b_{t}<0$ for all $t \in S$. If $S=\emptyset$, then it is clear that $(\bar{x},-1, \varepsilon) \in F_{n+2}$ for any $\varepsilon \in\left[-1,0\left[\right.\right.$. Otherwise, if $S \neq \emptyset$, let $r_{t}:=\left\langle a_{t}, \bar{x}\right\rangle-b_{t}<0$ for every $t \in S$. Since $S$ is finite, then $r:=\max _{t \in S}\left\{r_{t}\right\}<0$. By choosing $\varepsilon$ such that $\max \{-1, r\} \leq \varepsilon<0$, one has that

$$
\left\langle a_{t}, \bar{x}\right\rangle-b_{t}-\varepsilon \leq\left\langle a_{t}, \bar{x}\right\rangle-b_{t}-r \leq\left\langle a_{t}, \bar{x}\right\rangle-b_{t}-r_{t}=0
$$

for all $t \in S$, and so $(\bar{x},-1, \varepsilon) \in F_{n+2}$. The converse statement follows easily.

(ii) Now assume that $z<0$ for some $(x, y, z) \in F_{n+2}$. As $F_{n+2}$ is a cone and $y \leq$ $z<0$, then $-y>0$ and so $\frac{1}{-y}(x, y, z)=\left(\frac{x}{-y},-1, \frac{z}{-y}\right) \in F_{n+2}$ with $\frac{z}{-y} \in[-1,0[$. Then, in virtue of $(i), \frac{x}{-y} \in P$ and so $P \neq \emptyset$. The converse statement is a straightforward consequence of $(i)$.

As a particular case, the above lemma holds true whenever $S=\emptyset$ in (1). Thus, we recover the following result for finite linear systems containing only weak inequalities (see [9, Lemma 3.2]).

Corollary 3.6 Let $P \subset \mathbb{R}^{n}$ be the solution set of system $\sigma$ in (1) and assume that $S=\emptyset$. The following statements hold:

(i) If $P \neq \emptyset$, then $\bar{x} \in P$ if and only if $(\bar{x},-1) \in F_{n+1}$.

(ii) $P=\emptyset$ if and only if $y=0$ for all $(x, y) \in F_{n+1}$. 
Proof. (i) Assume that $P \neq \emptyset$ and let $\bar{x} \in P$. Then, $(\bar{x},-1) \in F_{n+1}$ follows from Lemma $3.5(i)$ and Lemma $3.3(i v)$. Conversely, if $(\bar{x},-1) \in F_{n+1}$, one has $(\bar{x},-1, \varepsilon) \in$ $F_{n+2}$ for any $\varepsilon \in[-1,0[$ as there are no strict inequalities, and so, by applying again Lemma $3.5(i), \bar{x} \in P$.

(ii) It is a straightforward consequence of $(i)$.

The main result in this section is a characterization of the consistency of a finite linear system containing strict inequalities in terms of its associated extended characteristic cone $\mathcal{K}$ introduced in Definition 3.2.

Theorem 3.7 Let $\sigma$ be the system in (1). Then, $\sigma$ is consistent if and only if $\left(0_{n}, 0,-1\right) \notin$ $\mathcal{K}$.

Proof. Firstly, assume that $\sigma$ is consistent and let $\bar{x} \in P$. By Lemma $3.5(i)$, there exists $\varepsilon \in\left[-1,0\left[\operatorname{such}\right.\right.$ that $(\bar{x},-1, \varepsilon) \in F_{n+2}$. If $\left(0_{n}, 0,-1\right) \in \mathcal{K}=\mathcal{K}^{\circ \circ}=F_{n+2}^{\circ}$, then $\left\langle\left(0_{n}, 0,-1\right),(\bar{x},-1, \varepsilon)\right\rangle \leq 0$ which implies $\varepsilon \geq 0$, and so, a contradiction. Thus, $\left(0_{n}, 0,-1\right) \notin \mathcal{K}$.

Conversely, if $\sigma$ is inconsistent, that is, $P=\emptyset$, then $z=0$ for all $(x, y, z) \in F_{n+2}$ according to Lemma $3.5(i i)$. As $\left\langle\left(0_{n}, 0,-1\right),(x, y, z)\right\rangle=0$ for all $(x, y, z) \in F_{n+2}$, one has $\left(0_{n}, 0,-1\right) \in F_{n+2}^{\circ}=\mathcal{K}$.

Corollary 3.8 ( $[30$, Theorem 1]) Let $\sigma$ be the system in $(1)$ and assume that $S=\emptyset$. Then, $\sigma$ is consistent if and only if $\left(0_{n},-1\right) \notin K$.

Proof. According to Theorem 3.7, it will be enough to prove that $\left(0_{n}, 0,-1\right) \in \mathcal{K}$ if and only if $\left(0_{n},-1\right) \in K$.

On the one hand, if $\left(0_{n},-1\right) \in K$, then $\left(0_{n},-1\right)=\sum_{t \in W} \lambda_{t}\left(a_{t}, b_{t}\right)+\alpha\left(0_{n}, 1\right)$ for some $\lambda_{t} \geq 0$ for all $t \in W$ and $\alpha \geq 0$. Hence, one has

$$
\left(0_{n}, 0,-1\right)=\sum_{t \in W} \lambda_{t}\left(a_{t}, b_{t}, 0\right)+(1+\alpha)\left(0_{n}, 1,-1\right)+\alpha\left(0_{n}, 0,1\right)
$$

and so $\left(0_{n}, 0,-1\right) \in \mathcal{K}$. Conversely, if $\left(0_{n}, 0,-1\right) \in \mathcal{K}$, then one has

$$
\left(0_{n}, 0,-1\right)=\sum_{t \in W} \lambda_{t}\left(a_{t}, b_{t}, 0\right)+\alpha\left(0_{n}, 1,-1\right)+\beta\left(0_{n}, 0,1\right)
$$

for some $\lambda_{t} \geq 0$ for all $t \in W, \alpha \geq 0$ and $\beta \geq 0$. As $\alpha=\beta+1$, one may write $\left(0_{n},-1\right)=\sum_{t \in W} \lambda_{t}\left(a_{t}, b_{t}\right)+\beta\left(0_{n}, 1\right)$ and so $\left(0_{n},-1\right) \in K$.

The following result characterizing the consistency of finite linear inequality systems is a reformulation of the characterization given in $[10$, Proposition 2.1] (in the finite case) where conditions (3) and (4) there are summarized here in condition (2) below.

Corollary 3.9 Let $\sigma$ be the system in (1). Then, $\sigma$ is consistent if and only if

$$
0_{n+1} \notin \operatorname{conv}\left\{\left(a_{t}, b_{t}\right), t \in S ;\left(0_{n}, 1\right)\right\}+\operatorname{cone}\left\{\left(a_{t}, b_{t}\right), t \in W\right\} .
$$


Proof. According to Theorem 3.7, it will be enough to prove that $\left(0_{n}, 0,-1\right) \in \mathcal{K}$ if and only if (2) does not hold.

On the one hand, if (2) does not hold, then

$$
\left(0_{n}, 0\right)=\sum_{t \in W} \lambda_{t}\left(a_{t}, b_{t}\right)+\sum_{t \in S} \mu_{t}\left(a_{t}, b_{t}\right)+\alpha\left(0_{n}, 1\right)
$$

for some $\lambda_{t} \geq 0$ for all $t \in W, \mu_{t} \geq 0$ for all $t \in S, \alpha \geq 0$ and $\sum_{t \in S} \mu_{t}+\alpha=1$. Hence, one has

$$
\left(0_{n}, 0,-1\right)=\sum_{t \in W} \lambda_{t}\left(a_{t}, b_{t}, 0\right)+\sum_{t \in S} \mu_{t}\left(a_{t}, b_{t},-1\right)+\alpha\left(0_{n}, 1,-1\right)+\beta\left(0_{n}, 0,1\right)
$$

with $\beta=0$, and so $\left(0_{n}, 0,-1\right) \in \mathcal{K}$.

Conversely, if $\left(0_{n}, 0,-1\right) \in \mathcal{K}$, one has (3) for some $\lambda_{t} \geq 0$ for all $t \in W, \mu_{t} \geq 0$ for all $t \in S, \alpha \geq 0$ and $\beta \geq 0$. Then, it easily follows that $\sum_{t \in S} \mu_{t}+\alpha=1+\beta \geq 1$. Hence, we may write

$$
\left(0_{n}, 0,-1\right)=\sum_{t \in W} \frac{\lambda_{t}}{1+\beta}\left(a_{t}, b_{t}, 0\right)+\sum_{t \in S} \frac{\mu_{t}}{1+\beta}\left(a_{t}, b_{t},-1\right)+\frac{\alpha}{1+\beta}\left(0_{n}, 1,-1\right)
$$

which implies $0_{n+1} \in \operatorname{conv}\left\{\left(a_{t}, b_{t}\right), t \in S ;\left(0_{n}, 1\right)\right\}+\operatorname{cone}\left\{\left(a_{t}, b_{t}\right), t \in W\right\}$.

The above corollary has important implications. Firstly, one has that, whenever $S=\emptyset, \sigma$ is consistent if and only if $\left(0_{n},-1\right) \notin$ cone $\left\{\left(a_{t}, b_{t}\right), t \in W\right\}$, and this is indeed equivalent to $\left(0_{n},-1\right) \notin K$ according to Corollary 3.8 (cf. $\lfloor 11$, Lemma 4.1]). Secondly, from this result it is easy to prove Motzkin transposition theorem [19]. And finally, we recover in Corollary 3.10 Motzkin theorem for the consistency of homogeneous linear systems containing strict inequalities (cf. [11, Theorem 3.5]).

Corollary 3.10 Let $\sigma_{0}$ be the homogeneous system associated to $\sigma$ in (1) obtained by letting $b_{t}=0$ for all $t \in T$. Then, $\sigma_{0}$ is consistent if and only if

$$
0_{n} \notin \operatorname{conv}\left\{a_{t}, t \in S\right\}+\operatorname{cone}\left\{a_{t}, t \in W\right\} .
$$

Proof. According to Corollary 3.9, $\sigma_{0}$ is consistent if and only if

$$
0_{n+1} \notin \operatorname{conv}\left\{\left(a_{t}, 0\right), t \in S ;\left(0_{n}, 1\right)\right\}+\operatorname{cone}\left\{\left(a_{t}, 0\right), t \in W\right\} .
$$

We just need to show that (4) and (5) are equivalent. If (4) holds, then clearly (5) also holds. Conversely, if (4) fails, then $0_{n}=\sum_{t \in S} \mu_{t} a_{t}+\sum_{t \in W} \lambda_{t} a_{t}$ for certain $\mu_{t} \geq 0$ (for all $t \in S$ ) and $\lambda_{t} \geq 0$ (for all $t \in W$ ) such that $\sum_{t \in S} \mu_{t}=1$. Thus, by letting $\alpha=0$ one has $0_{n+1}=\sum_{t \in S} \mu_{t}\left(a_{t}, b_{t}\right)+\alpha\left(0_{n}, 1\right)+\sum_{t \in W} \lambda_{t}\left(a_{t}, b_{t}\right)$ and so, (5) also fails.

\subsection{Consequence relations}

In this section we provide dual characterizations of linear inequalities which are consequence of a consistent finite linear system containing strict inequalities in terms of its associated extended characteristic cone. Thence, hereafter we shall assume that $(a, b) \in \mathbb{R}^{n} \times \mathbb{R}$ and that $\sigma$ in (1) is consistent. 
Proposition 3.11 Let $\sigma$ be the system in (1). Then, $\langle a, x\rangle \leq b$ is a consequence of $\sigma$ if and only if $(a, b, 0) \in \mathcal{K}$.

Proof. Assume that $(a, b, 0) \in \mathcal{K}=F_{n+2}^{\circ}$. Then, $\langle(a, b, 0),(x, y, z)\rangle \leq 0$ for all $(x, y, z) \in F_{n+2}$. According to Lemma 3.5, for every $x \in P$ there exists $\varepsilon \in[-1,0[$ such that $(x,-1, \varepsilon) \in F_{n+2}$. Hence, $\langle(a, b, 0),(x,-1, \varepsilon)\rangle \leq 0$ and so $\langle a, x\rangle \leq b$ for all $x \in P$.

Now assume that $\langle a, x\rangle \leq b$ is a consequence of $\sigma$. We shall prove that $(a, b, 0) \in$ $F_{n+2}^{\circ}$. For that purpose, given $(x, y, z) \in F_{n+2}$, we distinguish two cases:

Case 1: $y \leq z<0$. It is easy to see that $\left(\frac{x}{-y},-1, \frac{z}{-y}\right) \in F_{n+2}$ with $-1 \leq \frac{z}{-y}<0$, and so $x^{*}:=\frac{x}{-y} \in P$. By hypothesis, one has $\left\langle a, x^{*}\right\rangle \leq b$, that is, $\langle a, x\rangle+b y \leq 0$.

Case 2: $y \leq z=0$. Let $\bar{x} \in P$ and $\varepsilon \in\left[-1,0\left[\right.\right.$ such that $(\bar{x},-1, \varepsilon) \in F_{n+2}$. Then,

$$
\left(x_{\lambda}, y_{\lambda}, z_{\lambda}\right):=(1-\lambda)(x, y, 0)+\lambda(\bar{x},-1, \varepsilon) \in F_{n+2}
$$

for all $\lambda \in] 0,1\left[\right.$ since $F_{n+2}$ is convex. As $y_{\lambda} \leq z_{\lambda}<0$, the vector $\left(x_{\lambda}, y_{\lambda}, z_{\lambda}\right) \in F_{n+2}$ corresponds to Case 1. Thus,

$$
(1-\lambda)(\langle a, x\rangle+b y)+\lambda(\langle a, \bar{x}\rangle-b)=\left\langle a, x_{\lambda}\right\rangle+b y_{\lambda} \leq 0
$$

for all $\lambda \in] 0,1[$. By taking limits when $\lambda \rightarrow 0$, one has $\langle a, x\rangle+b y \leq 0$.

Corollary 3.12 (Farkas-Minkowski Theorem) Let $\bar{\sigma}$ be the relaxed system of $\sigma$. Then, $\langle a, x\rangle \leq b$ is a consequence of $\bar{\sigma}$ if and only if $(a, b) \in K$.

Proof. It easily follows from the fact that $\langle a, x\rangle \leq b$ is a consequence of $\sigma$ if only if it is a consequence of $\bar{\sigma}$, and by applying Proposition 3.11 and Lemma 3.3 (iii).

If we denote by $K \leq$ the weak dual cone of $P$ (the solution set of $\sigma$ ), then the above results guarantee the identities

$$
K^{\leq}=\left\{(a, b) \in \mathbb{R}^{n} \times \mathbb{R}:(a, b, 0) \in \mathcal{K}\right\}=\pi\left(\mathcal{K} \cap\left(\mathbb{R}^{n+1} \times\{0\}\right)\right)=\pi(\mathcal{K})=K .
$$

Similarly to Proposition 3.11, next result characterizes the strict inequalities which are consequence of the linear system $\sigma$.

Proposition 3.13 Let $\sigma$ be the system in (1). Then, $\langle a, x\rangle\langle b$ is a consequence of $\sigma$ if and only if $(a, b, c) \in \mathcal{K}$ for some $c<0$.

Proof. Assume that $(a, b, c) \in \mathcal{K}=F_{n+2}^{\circ}$ for some $c<0$. Then, $\langle(a, b, c),(x, y, z)\rangle \leq 0$ for all $(x, y, z) \in F_{n+2}$. Given $\bar{x} \in P$, according to Lemma 3.5 there exists $\varepsilon \in[-1,0[$ such that $(\bar{x},-1, \varepsilon) \in F_{n+2}$. Hence, $\langle(a, b, c),(\bar{x},-1, \varepsilon)\rangle \leq 0$ and so $\langle a, \bar{x}\rangle-b+c \varepsilon \leq 0$. As $c \varepsilon>0$, one has $\langle a, \bar{x}\rangle-b<0$. Thus, $\langle a, x\rangle<b$ holds for all $x \in P$.

Now assume that $\langle a, x\rangle<b$ is a consequence of $\sigma$. This means that the linear system $\sigma \cup\{\langle-a, x\rangle \leq-b\}$ is inconsistent and so, by Theorem 3.7, $\left(0_{n}, 0,-1\right)$ belongs to its associated extended characteristic cone, that is,

$$
\left(0_{n}, 0,-1\right)=\sum_{t \in W} \lambda_{t}\left(a_{t}, b_{t}, 0\right)+\alpha(-a,-b, 0)+\sum_{t \in S} \mu_{t}\left(a_{t}, b_{t},-1\right)+\beta\left(0_{n}, 1,-1\right)+\gamma\left(0_{n}, 0,1\right)
$$


for some $\lambda_{t} \geq 0$ for all $t \in W, \mu_{t} \geq 0$ for all $t \in S$ and $\alpha, \beta, \gamma \geq 0$. Since $\alpha>0$ (otherwise $\sigma$ would be inconsistent), we may write

$$
\left(a, b, \frac{-1}{\alpha}\right)=\sum_{t \in W} \frac{\lambda_{t}}{\alpha}\left(a_{t}, b_{t}, 0\right)+\sum_{t \in S} \frac{\mu_{t}}{\alpha}\left(a_{t}, b_{t},-1\right)+\frac{\beta}{\alpha}\left(0_{n}, 1,-1\right)+\frac{\gamma}{\alpha}\left(0_{n}, 0,1\right)
$$

Hence, $(a, b, c) \in \mathcal{K}$ with $c:=\frac{-1}{\alpha}<0$.

Observe that, as stated in the proof of the above proposition, an inequality $\langle a, x\rangle<b$ is a consequence of $\sigma$ if and only if the system $\sigma \cup\{\langle-a, x\rangle \leq-b\}$ is inconsistent. Thus, by applying Corollary 3.9, one has that $\langle a, x\rangle<b$ is a consequence of $\sigma$ if and only if

$$
0_{n+1} \in \operatorname{conv}\left\{\left(a_{t}, b_{t}\right), t \in S ;\left(0_{n}, 1\right)\right\}+\operatorname{cone}\left\{\left(a_{t}, b_{t}\right), t \in W ;-(a, b)\right\} .
$$

If we denote by $K^{<}$the strict dual cone of $P$, then Proposition 3.13 establishes

$$
K^{<}=\left\{(a, b) \in \mathbb{R}^{n} \times \mathbb{R}:(a, b, c) \in \mathcal{K} \text { for some } c<0\right\}=\pi\left(\mathcal{K} \cap\left(\mathbb{R}^{n+1} \times \mathbb{R}_{--}\right)\right)
$$

where $\mathbb{R}_{--}$stands for the set of negative real numbers.

The negative value $c$ in Proposition 3.13 depends on each strict inequality and it is not unique as $\left(0_{n}, 0,1\right) \in \operatorname{rec}(\mathcal{K})$. Thus, it follows that if $(a, b,-1) \in \mathcal{K}$ then $\langle a, x\rangle<b$ is a consequence of $\sigma$, but the converse statement is not true in general as it is shown in the following example.

Example 3.14 Let $\sigma$ be the system in Example 3.4. The strict inequality $-2 x_{1}-x_{2}<$ -3 is a consequence of $\sigma$, but $(-2,-1,-3,-1) \notin \mathcal{K}$. Nevertheless, $\left(-2,-1,-3,-\frac{1}{2}\right) \in$ $\mathcal{K}$ and so the characterization in Proposition 3.13 holds.

Inspired by [16], a strict inequality $\langle a, x\rangle<b$ is said to be a legal linear combination of $\sigma$ if one can write

$$
(a, b)=\sum_{t \in W} \lambda_{t}\left(a_{t}, b_{t}\right)+\sum_{t \in S} \mu_{t}\left(a_{t}, b_{t}\right)
$$

with $\lambda_{t} \geq 0$ for all $t \in W$ and $\mu_{t} \geq 0$ for all $t \in S$ such that $\sum_{t \in S} \mu_{t}>0$.

Corollary 3.15 Let $\sigma$ be the system in (1). Then, $\langle a, x\rangle<b$ is a consequence of $\sigma$ if and only if $\langle a, x\rangle<b$ is a legal linear combination of $\sigma \cup\left\{\left\langle 0_{n}, x\right\rangle<1\right\}$.

Proof. According to Proposition 3.13, it will be enough to prove that $(a, b, c) \in \mathcal{K}$ for some $c<0$ if and only if $\langle a, x\rangle<b$ is a legal linear combination of $\sigma \cup\left\{\left\langle 0_{n}, x\right\rangle<1\right\}$.

On the one hand, if $(a, b, c) \in \mathcal{K}$ for some $c<0$, then

$$
(a, b, c)=\sum_{t \in W} \lambda_{t}\left(a_{t}, b_{t}, 0\right)+\sum_{t \in S} \mu_{t}\left(a_{t}, b_{t},-1\right)+\gamma\left(0_{n}, 1,-1\right)+\alpha\left(0_{n}, 0,1\right)
$$

with $\lambda_{t} \geq 0$ for all $t \in W, \mu_{t} \geq 0$ for all $t \in S, \gamma \geq 0$ and $\alpha \geq 0$. Hence, one has

$$
(a, b)=\sum_{t \in W} \lambda_{t}\left(a_{t}, b_{t}\right)+\sum_{t \in S} \mu_{t}\left(a_{t}, b_{t}\right)+\gamma\left(0_{n}, 1\right)
$$


with $\sum_{t \in S} \mu_{t}+\gamma \geq \sum_{t \in S} \mu_{t}+\gamma-\alpha=-c>0$ which means that $\langle a, x\rangle<b$ is a legal linear combination of $\sigma \cup\left\{\left\langle 0_{n}, x\right\rangle<1\right\}$.

On the other hand, if $\langle a, x\rangle<b$ is a legal linear combination of $\sigma \cup\left\{\left\langle 0_{n}, x\right\rangle<1\right\}$, then one has

$$
(a, b)=\sum_{t \in W} \lambda_{t}\left(a_{t}, b_{t}\right)+\sum_{t \in S} \mu_{t}\left(a_{t}, b_{t}\right)+\gamma\left(0_{n}, 1\right)
$$

with $\lambda_{t} \geq 0$ for all $t \in W, \mu_{t} \geq 0$ for all $t \in S$ and $\gamma \geq 0$ such that $\beta:=\sum_{t \in S} \mu_{t}+\gamma>0$. Hence, one can write

$$
\left(a, b, \frac{-\beta}{2}\right)=\sum_{t \in W} \lambda_{t}\left(a_{t}, b_{t}, 0\right)+\sum_{t \in S} \mu_{t}\left(a_{t}, b_{t},-1\right)+\gamma\left(0_{n}, 1,-1\right)+\frac{\beta}{2}\left(0_{n}, 0,1\right) .
$$

Thus, $(a, b, c) \in \mathcal{K}$ with $c:=\frac{-\beta}{2}<0$, and the conclusion follows.

As a consequence of Proposition 3.13 we obtain the classical characterization of a strict inequality which is a consequence of a consistent finite system containing only weak inequalities (cf. $\mid 12$, Proposition 1.2]).

Corollary 3.16 Let $\sigma$ be the system in (1) and assume that $S=\emptyset$. Then, $\langle a, x\rangle<b$ is a consequence of $\sigma$ if and only if $(a, b) \in K$ and $\left(0_{n},-1\right) \in K+\operatorname{span}\{(a, b)\}$.

Proof. According to Proposition 3.13, it will be enough to prove that $(a, b, c) \in \mathcal{K}$ for some $c<0$ if and only if $(a, b) \in K$ and $\left(0_{n},-1\right) \in K+\operatorname{span}\{(a, b)\}$.

On the one hand, if $(a, b, c) \in \mathcal{K}$ for some $c<0$, then

$$
(a, b, c)=\sum_{t \in W} \lambda_{t}\left(a_{t}, b_{t}, 0\right)+\alpha\left(0_{n}, 1,-1\right)+\beta\left(0_{n}, 0,1\right)
$$

for some $\lambda_{t} \geq 0$ for all $t \in W, \alpha \geq 0$ and $\beta \geq 0$. Clearly, one has $(a, b) \in K$ and $c=-\alpha+\beta<0$, which implies $0 \leq \beta<\alpha$. Hence, we may write

$$
\left(0_{n},-1,0\right)=\sum_{t \in W} \frac{\lambda_{t}}{\alpha}\left(a_{t}, b_{t}, 0\right)-\frac{1}{\alpha}(a, b, 0)
$$

and so, $\left(0_{n},-1\right) \in K+\operatorname{span}\{(a, b)\}$.

On the other hand, if $(a, b) \in K$ and $\left(0_{n},-1\right) \in K+\operatorname{span}\{(a, b)\}$, then one has

$$
\left(0_{n},-1\right)=\sum_{t \in W} \lambda_{t}\left(a_{t}, b_{t}\right)+\alpha\left(0_{n}, 1\right)+\beta(a, b)
$$

for some $\lambda_{t} \geq 0$ for all $t \in W, \alpha \geq 0$ and $\beta \in \mathbb{R}$. If $\beta=0$ then $\left(0_{n},-1\right) \in K$ and $\sigma$ would be inconsistent. If $\beta>0$, as $(a, b) \in K$, we obtain $\left(0_{n},-1\right) \in K$ again. Hence, $\beta<0$ and, by letting $c:=\frac{1+\alpha}{\beta}<0$ and $\gamma:=0$, one has

$$
(a, b, c)=\sum_{t \in W} \frac{\lambda_{t}}{-\beta}\left(a_{t}, b_{t}, 0\right)+\frac{1+\alpha}{-\beta}\left(0_{n}, 1,-1\right)+\gamma\left(0_{n}, 0,1\right)
$$

and the conclusion follows. 


\subsection{Set containments}

As every e-polyhedron is an evenly convex set, one may apply the dual characterization stated in [8] to the set containment of e-polyhedra by means of their strict dual cones. Furthermore, if an external representation of an e-polyhedron is known, its strict dual cone can be obtained through its extended characteristic cone in virtue of (7).

Proposition 3.17 Let $P_{i} \subset \mathbb{R}^{n}$ be the solution set of the consistent finite system

$$
\sigma_{i}=\left\{\left\langle a_{t}^{i}, x\right\rangle \leq b_{t}^{i}, t \in W_{i} ;\left\langle a_{t}^{i}, x\right\rangle<b_{t}^{i}, t \in S_{i}\right\}
$$

whose extended characteristic cone is $\mathcal{K}_{i}$, for $i=1,2$. Then, $P_{1} \subset P_{2}$ if and only if $K_{2}^{<} \subset K_{1}^{<}$, where $K_{i}^{<}=\pi\left(\mathcal{K}_{i} \cap\left(\mathbb{R}^{n+1} \times \mathbb{R}_{--}\right)\right)$.

The above characterization fails if the strict dual cone is replaced by the weak dual cone as e-polyhedra are not necessarily closed. Indeed, if $K_{i}^{\leq}$is the weak dual cone of $P_{i}, i=1,2$, then $K_{2}^{\leq} \subset K_{1}^{\leq}$is a necessary (but not sufficient) condition for the set containment $P_{1} \subset P_{2}$.

Since the external representation of an e-polyhedron contains a finite number of both weak and strict inequalities, one wonders whether the extended characteristic cone associated to such a representation, which contains full information of both weak and strict dual cones, characterizes the containment of e-polyhedra.

Proposition 3.18 (Sufficient condition) Let $P_{i} \subset \mathbb{R}^{n}$ be the solution set of the consistent finite system $\sigma_{i}$ in (8) whose extended characteristic cone is $\mathcal{K}_{i}, i=1,2$. If $\mathcal{K}_{2} \subset \mathcal{K}_{1}$, then $P_{1} \subset P_{2}$.

Proof. Let $x \in P_{1}$. By Lemma 3.5, one has $(x,-1, \varepsilon) \in \mathcal{K}_{1}^{\circ}$ for some $\varepsilon \in[-1,0[$. As $\mathcal{K}_{2} \subset \mathcal{K}_{1}$, then $\mathcal{K}_{1}^{\circ} \subset \mathcal{K}_{2}^{\circ}$. Hence, $(x,-1, \varepsilon) \in \mathcal{K}_{2}^{\circ}$ and so $x \in P_{2}$ by applying again Lemma 3.5.

The converse statement does not hold in general as we illustrate in the next example.

Example 3.19 Let consider the systems $\sigma_{1}=\left\{-x_{1}-x_{2} \leq-2,-x_{1}+x_{2}<0\right\}$ and $\sigma_{2}=\left\{-2 x_{1}-x_{2}<-3\right\}$ in $\mathbb{R}^{2}$. One has that $P_{1} \subset P_{2}$, however $\mathcal{K}_{2} \nsubseteq \mathcal{K}_{1}$ as $(-2,-1,-3,-1) \in \mathcal{K}_{2} \backslash \mathcal{K}_{1}$ (see Example 3.14).

The fact that the extended characteristic cone does not characterize the set containment of e-polyhedra relies on its definition. In contrast with the characteristic cone of a polyhedron, which does not depend on its external representation, the extended characteristic cone depends on the finite linear inequality system defining an e-polyhedron. Hence, a given e-polyhedron can be written as the solution set of two different finite linear inequality systems and their associated extended characteristic cones may not coincide. Thus, if $\sigma$ in (1) is an external representation of an e-polyhedron $P$, we observe that if we replace a weak inequality $\langle a, x\rangle \leq b$ in $\sigma$ by the equivalent one $\langle\alpha a, x\rangle \leq \alpha b$ (with $\alpha>0$ ), then the extended characteristic cone does not change as $(a, b, 0) \in \mathcal{K}$ if and only if $(\alpha a, \alpha b, 0) \in \mathcal{K}$. However, if we replace a strict inequality $\langle a, x\rangle<b$ in $\sigma$ by the equivalent one $\langle\alpha a, x\rangle\langle\alpha b$ (with $\alpha>0$ ), then the extended characteristic cone may change as $(\alpha a, \alpha b,-1)$ does not necessarily belong to $\mathcal{K}$. Next result provides more details on the extended characteristic cones of equivalent systems. 
Lemma 3.20 Let $\sigma$ be the system in (1). Let $\sigma^{\star}$ be the system $\sigma$ where the strict inequalities of indices in $S^{\star} \subset S$ have been equivalently replaced by $\left\langle\alpha_{t} a_{t}, x\right\rangle<\alpha_{t} b_{t}$ for certain $\alpha_{t}>0$ for all $t \in S^{\star}$. Let $\mathcal{K}$ and $\mathcal{K}^{\star}$ be the extended characteristic cones associated to $\sigma$ and $\sigma^{\star}$, respectively. The following statements hold:

(i) If $\alpha_{t}>1$ for all $t \in S^{\star}$, then $\mathcal{K}^{\star} \subset \mathcal{K}$.

(ii) If $\alpha_{t}<1$ for all $t \in S^{\star}$, then $\mathcal{K} \subset \mathcal{K}^{\star}$.

Proof. (i) One has that $\mathcal{K}^{\star}$ is the cone finitely generated by

$$
\left\{\left(a_{t}, b_{t}, 0\right), t \in W ;\left(a_{t}, b_{t},-1\right), t \in S \backslash S^{\star} ;\left(\alpha_{t} a_{t}, \alpha_{t} b_{t},-1\right), t \in S^{\star} ;\left(0_{n}, 1,-1\right) ;\left(0_{n}, 0,1\right)\right\} .
$$

Hence, it will be enough to prove that $\left(\alpha_{t} a_{t}, \alpha_{t} b_{t},-1\right) \in \mathcal{K}$ for all $t \in S^{\star}$. Given $t \in S^{\star}$, as $\alpha_{t}>1$, one may write

$$
\left(\alpha_{t} a_{t}, \alpha_{t} b_{t},-1\right)=\alpha_{t}\left(a_{t}, b_{t}, \frac{-1}{\alpha_{t}}\right)=\alpha_{t}\left(a_{t}, b_{t},-1\right)+\left(\alpha_{t}-1\right)\left(0_{n}, 0,1\right)
$$

and so $\left(\alpha_{t} a_{t}, \alpha_{t} b_{t},-1\right) \in \mathcal{K}$.

(ii) It is a straightforward consequence of $(i)$ by interchanging the role of systems $\sigma$ and $\sigma^{\star}$.

Thus, given a finite linear system, we may obtain easily an equivalent system such that its extended characteristic cone is either a contraction or an enlargement of the one associated to the original system. Nevertheless, all the properties of e-polyhedra studied in this section, as equations (6) and (7), for instance, hold for any external representation chosen.

Next we provide two necessary conditions for the containment of e-polyhedra in terms of our new dual cone.

Proposition 3.21 (Necessary conditions) Let $P_{i} \subset \mathbb{R}^{n}$ be the solution set of the consistent finite system $\sigma_{i}$ in (8) whose extended characteristic cone is $\mathcal{K}_{i}$, for $i=1,2$. If $P_{1} \subset P_{2}$, then the following statements hold:

(i) $\mathcal{K}_{2}^{\star} \subset \mathcal{K}_{1}$ for some external representation $\sigma_{2}^{\star}$ of $P_{2}$ with extended characteristic cone $\mathcal{K}_{2}^{\star}$. Moreover, one has $\mathcal{K}_{2}^{\star} \subset \mathcal{K}_{2}$.

(ii) $\mathcal{K}_{2} \subset \mathcal{K}_{1}^{\star}$ where $\mathcal{K}_{1}^{\star}$ is the extended characteristic cone associated to the external representation of $P_{1}$ given by $\sigma_{1}^{\star}:=\sigma_{1} \cup \sigma_{2}$.

Proof. ( $i$ ) If $P_{1} \subset P_{2}$, then every inequality in $\sigma_{2}$ is consequence of $\sigma_{1}$.

On the one hand, given $t \in W_{2}$, as $\left\langle a_{t}^{2}, x\right\rangle \leq b_{t}^{2}$ is a consequence of $\sigma_{1}$ then $\left(a_{t}^{2}, b_{t}^{2}, 0\right) \in \mathcal{K}_{1}$ in virtue of Proposition 3.11. On the other hand, given $t \in S_{2}$, as $\left\langle a_{t}^{2}, x\right\rangle<b_{t}^{2}$ is a consequence of $\sigma_{1}$ then $\left(a_{t}^{2}, b_{t}^{2}, c_{t}\right) \in \mathcal{K}_{1}$ for some $c_{t}<0$ in virtue of Proposition 3.13. Let $S_{2}^{\star}$ be the set of indices $t \in S_{2}$ such that $\left(a_{t}^{2}, b_{t}^{2}, c_{t}\right) \in \mathcal{K}_{1}$ for some $-1<c_{t}<0$. We now distinguish two cases. 
- If $t \in S_{2} \backslash S_{2}^{\star}$, then $\left(a_{t}^{2}, b_{t}^{2}, c_{t}\right) \in \mathcal{K}_{1}$ for some $c_{t} \leq-1$. As $\left(0_{n}, 0,1\right) \in \operatorname{rec}\left(\mathcal{K}_{1}\right)$, one has $\left(a_{t}^{2}, b_{t}^{2},-1\right) \in \mathcal{K}_{1}$.

- If $t \in S_{2}^{\star}$, as $\mathcal{K}_{1}$ is a cone and $\frac{1}{-c_{t}}>0$, then $\left(\frac{a_{t}^{2}}{-c_{t}}, \frac{b_{t}^{2}}{-c_{t}},-1\right) \in \mathcal{K}_{1}$.

Hence, one has $\mathcal{K}_{2}^{\star} \subset \mathcal{K}_{1}$ being $\mathcal{K}_{2}^{\star}$ the associated extended characteristic cone associated to the following external representation of $P_{2}$,

$$
\sigma_{2}^{\star}=\left\{\left\langle a_{t}^{2}, x\right\rangle \leq b_{t}^{2}, t \in W_{2} ;\left\langle a_{t}^{2}, x\right\rangle<b_{t}^{2}, t \in S_{2} \backslash S_{2}^{\star} ;\left\langle\frac{a_{t}^{2}}{-c_{t}}, x\right\rangle<\frac{b_{t}^{2}}{-c_{t}}, t \in S_{2}^{\star}\right\} .
$$

Last statement follows from Lemma 3.20 as $\frac{1}{-c_{t}}>1$ for all $t \in S_{2}^{\star}$.

(ii) If $P_{1} \subset P_{2}$, then $\sigma_{1}^{\star}:=\sigma_{1} \cup \sigma_{2}$ is an external representation of $P_{1}$ and so, every inequality in $\sigma_{2}$ is consequence of $\sigma_{1}^{\star}$. As any generator of $\mathcal{K}_{2}$ is also a generator of $\mathcal{K}_{1}^{\star}$, it easily follows that $\mathcal{K}_{2} \subset \mathcal{K}_{1}^{\star}$.

Proposition 3.22 Let $P=\bigcap_{i=1}^{m} P_{i} \neq \emptyset$, where $P_{i} \subset \mathbb{R}^{n}$ is the solution set of the system $\sigma_{i}$ in (8) whose extended characteristic cone is $\mathcal{K}_{i}$, for $i=1, \ldots, m$. If $\mathcal{K}$ is the extended characteristic cone of $\sigma:=\cup_{i=1}^{m} \sigma_{i}$ (an external representation of $P$ ), then

$$
\mathcal{K}=\operatorname{clconv}\left(\bigcup_{i=1}^{m} \mathcal{K}_{i}\right)
$$

Proof. As $P \subset P_{i}$ for all $i=1, \ldots, m$, Proposition 3.21 yields $\mathcal{K}_{i} \subset \mathcal{K}$ for all $i=1, \ldots, m$, and so $\operatorname{clconv}\left(\bigcup_{i=1}^{m} \mathcal{K}_{i}\right) \subset \mathcal{K}$.

Assume that there exists $(\bar{x}, \bar{y}, \bar{z}) \in \mathcal{K} \backslash \operatorname{cl} \operatorname{conv}\left(\bigcup_{i=1}^{m} \mathcal{K}_{i}\right)$. Then, there exists $(a, b, c) \in$ $\mathbb{R}^{n+2}$ such that $\langle(a, b, c),(x, y, z)\rangle \leq 0<\langle(a, b, c),(\bar{x}, \bar{y}, \bar{z})\rangle$ for all $(x, y, z) \in \bigcup_{i=1}^{m} \mathcal{K}_{i}$, which implies that $(a, b, c) \in \mathcal{K}^{\circ}$. In that case, one has $\langle(a, b, c),(\bar{x}, \bar{y}, \bar{z})\rangle \leq 0$ which entails a contradiction.

\section{Representation of e-polyhedra}

In this section we shall obtain an internal representation of an e-polyhedron by using the extended characteristic cone associated to an external representation of such an e-polyhedron. Thus, we prove a generalization of Motzkin theorem for a larger family of polyhedral sets. We start by extending the notion of polytope.

Definition 4.1 A subset $Q \subset \mathbb{R}^{n}$ is said to be an evenly convex polytope (e-polytope, in brief) if there are two finite sets $U:=\left\{u_{1}, \ldots, u_{m}\right\}$ and $V:=\left\{v_{1}, \ldots, v_{q}\right\}$ in $\mathbb{R}^{n}$ such that

$$
Q=\left\{\sum_{i=1}^{m} \lambda_{i} u_{i}+\sum_{j=1}^{q} \mu_{j} v_{j}: \lambda \in \mathbb{R}_{+}^{m}, \mu \in \mathbb{R}_{+}^{q}, \sum_{i=1}^{m} \lambda_{i}+\sum_{j=1}^{q} \mu_{j}=1, \sum_{i=1}^{m} \lambda_{i}>0\right\}
$$

In short, we shall denote $Q=\operatorname{conv}(U \mid V)$. 
Each point of $Q$ can be thought as a kind of legal convex combination, that is, a convex combination of finitely many points in $U \cup V$ with a positive weight associated to at least one point in $U$. It easily follows from the definition that $Q$ contains the set $U$, but it does not contain $V$ necessarily. Moreover, one has $\operatorname{conv}(U \mid V) \neq \operatorname{conv}(V \mid U)$, in general, although $\operatorname{conv}(U \mid \emptyset)=\operatorname{conv}(\emptyset \mid U)=\operatorname{conv}(U)$. Thus, if either $U$ or $V$ are empty, then $Q$ becomes a polytope in the classical sense.

Next result shows that the closure of an e-polytope is, in fact, a polytope which contains the set $U \cup V$.

Lemma 4.2 Let $Q=\operatorname{conv}(U \mid V)$ be an e-polytope as in (9). Then,

(i) $\operatorname{cl} Q=\operatorname{conv}(U \cup V)$.

(ii) $Q$ is wholefaced.

(iii) $Q$ is a bounded e-polyhedron.

Proof. (i) Observe that $Q \subset \operatorname{conv}(U \cup V)$ and $\operatorname{conv}(U \cup V)$ is a closed convex set. Thus, $\operatorname{cl} Q \subset \operatorname{conv}(U \cup V)$. Conversely, if $x \in \operatorname{conv}(U \cup V)$, there exist $\lambda \in \mathbb{R}_{+}^{m}, \mu \in \mathbb{R}_{+}^{q}$ such that $x=\sum_{i=1}^{m} \lambda_{i} u_{i}+\sum_{j=1}^{q} \mu_{j} v_{j}$ and $\sum_{i=1}^{m} \lambda_{i}+\sum_{j=1}^{q} \mu_{j}=1$. If $\sum_{i=1}^{m} \lambda_{i}>0$, then there is nothing to prove. Otherwise, one can write $x=\mu_{j_{0}} v_{j_{0}}+\sum_{j \neq j_{0}} \mu_{j} v_{j}$ for some $j_{0} \in\{1, \ldots, q\}$ such that $\mu_{j_{0}}>0$. By letting $0<\delta<\mu_{j_{0}}$ and defining $x^{\delta}:=\left(\mu_{j_{0}}-\delta\right) v_{j_{0}}+\sum_{j \neq j_{0}} \mu_{j} v_{j}+\delta u_{i_{0}}$ for some $i_{0} \in\{1, \ldots, m\}$, one has $x^{\delta} \in Q$ and $x=\lim _{\delta \rightarrow 0} x^{\delta} \in \operatorname{cl} Q$. Thus, $\operatorname{cl} Q=\operatorname{conv}(U \cup V)$.

(ii) Let $x^{1} \in Q$ and $x^{2} \in \operatorname{cl} Q$. By $(i)$, one can write $x^{k}=\sum_{i=1}^{m} \lambda_{i}^{k} u_{i}+\sum_{j=1}^{q} \mu_{j}^{k} v_{j}$ for certain $\lambda^{k} \in \mathbb{R}_{+}^{m}, \mu^{k} \in \mathbb{R}_{+}^{q}$ such that $\sum_{i=1}^{m} \lambda_{i}^{k}+\sum_{j=1}^{q} \mu_{j}^{k}=1$ (for $k=1,2$ ) and $\sum_{i=1}^{m} \lambda_{i}^{1}>0$. It is easy to see that $(1-\gamma) x^{1}+\gamma x^{2} \in Q$ for any $\gamma \in[0,1[$ and so, $Q$ is wholefaced.

(iii) Observe that $\operatorname{cl} Q$ is a polytope, and so a bounded polyhedron. It is wellknown (cf. [7]) that a wholefaced set whose closure is a polyhedron is an evenly convex polyhedral set. Hence, $Q$ is a bounded e-polyhedron.

Consequently, $Q$ is the result of eliminating from the polytope $\operatorname{cl} Q$ the union of a certain family of its faces. Next we characterize the two main particular cases, whenever $Q$ is closed and relatively open.

Proposition 4.3 Let $Q=\operatorname{conv}(U \mid V)$ be an e-polytope as in (9). Then,

(i) $Q$ is closed if and only if $V \subset Q$.

(ii) $Q$ is relatively open if and only if $U \subset \operatorname{rint} Q$.

Proof. ( $i$ ) If $Q$ is closed, then $Q=\operatorname{cl} Q=\operatorname{conv}(U \cup V) \supset V$.

Assume now that $V \subset Q$. We just need to show $\operatorname{cl} Q \subset Q$. Thus, if $x \in \operatorname{cl} Q$, there exist $\lambda \in \mathbb{R}_{+}^{m}, \mu \in \mathbb{R}_{+}^{q}$ such that $x=\sum_{i=1}^{m} \lambda_{i} u_{i}+\sum_{j=1}^{q} \mu_{j} v_{j}$ and $\sum_{i=1}^{m} \lambda_{i}+\sum_{j=1}^{q} \mu_{j}=1$. If $\sum_{i=1}^{m} \lambda_{i}>0$, then $x \in Q$. Otherwise, one has $x=\sum_{j=1}^{q} \mu_{j} v_{j}$ with $\sum_{j=1}^{q} \mu_{j}=1$. As 
$V \subset Q$, for every $j=1, \ldots, q$, there exist $\rho^{j} \in \mathbb{R}_{+}^{m}, \omega^{j} \in \mathbb{R}_{+}^{q}$ with $\sum_{k=1}^{m} \rho_{k}^{j}+\sum_{l=1}^{q} \omega_{l}^{j}=1$ and $\sum_{k=1}^{m} \rho_{k}^{j}>0$ such that $v_{j}=\sum_{k=1}^{m} \rho_{k}^{j} u_{k}+\sum_{l=1}^{q} \omega_{l}^{j} v_{l}$. Then,

$$
x=\sum_{j=1}^{q} \mu_{j}\left(\sum_{k=1}^{m} \rho_{k}^{j} u_{k}+\sum_{l=1}^{q} \omega_{l} v_{l}\right)=\sum_{k=1}^{m}\left(\sum_{j=1}^{q} \mu_{j} \rho_{k}^{j}\right) u_{k}+\sum_{l=1}^{q}\left(\sum_{j=1}^{q} \mu_{j} \omega_{l}^{j}\right) v_{l}
$$

with $\sum_{k=1}^{m} \sum_{j=1}^{q} \mu_{j} \rho_{k}^{j}>0$ as $\mu_{j}>0$ for at least one index $j$. Consequently, $x \in Q$.

(ii) If $Q$ is relatively open, then $\operatorname{rint} Q=Q \supset U$.

Assume now that $U \subset \operatorname{rint} Q$. We just need to show $Q \subset \operatorname{rint} Q$. Observe that

$$
\operatorname{rint} Q=\operatorname{rint}(\operatorname{cl} Q)=\left\{\sum_{i=1}^{m} \lambda_{i} u_{i}+\sum_{j=1}^{q} \mu_{j} v_{j}: \lambda \in \mathbb{R}_{++}^{m}, \mu \in \mathbb{R}_{++}^{q}, \sum_{i=1}^{m} \lambda_{i}+\sum_{j=1}^{q} \mu_{j}=1\right\},
$$

in virtue of $[21$, Theorem 6.3] and [5, Lemma 2.3]. Thus, if $x \in Q$, there exist $\lambda \in$ $\mathbb{R}_{+}^{m}, \mu \in \mathbb{R}_{+}^{q}$ such that $x=\sum_{i=1}^{m} \lambda_{i} u_{i}+\sum_{j=1}^{q} \mu_{j} v_{j}$ with $\sum_{i=1}^{m} \lambda_{i}+\sum_{j=1}^{q} \mu_{j}=1$ and $\sum_{i=1}^{m} \lambda_{i}>0$. As $U \subset \operatorname{rint} Q$, for every $i=1, \ldots, m$, there exist $\rho^{i} \in \mathbb{R}_{++}^{m}, \omega^{i} \in \mathbb{R}_{++}^{q}$ with $\sum_{k=1}^{m} \rho_{k}^{i}+\sum_{l=1}^{q} \omega_{l}^{i}=1$ such that $u_{i}=\sum_{k=1}^{m} \rho_{k}^{i} u_{k}+\sum_{l=1}^{q} \omega_{l}^{i} v_{l}$. Then,

$$
x=\sum_{i=1}^{m} \lambda_{i}\left(\sum_{k=1}^{m} \rho_{k}^{i} u_{k}+\sum_{l=1}^{q} \omega_{l}^{i} v_{l}\right)+\sum_{j=1}^{q} \mu_{j} v_{j}=\sum_{k=1}^{m}\left(\sum_{i=1}^{m} \lambda_{i} \rho_{k}^{i}\right) u_{k}+\sum_{l=1}^{q}\left(\mu_{l}+\sum_{i=1}^{m} \lambda_{i} \omega_{l}^{i}\right) v_{l}
$$

with $\sum_{i=1}^{m} \lambda_{i} \rho_{k}^{i}>0$ for every $k=1, \ldots, m$ and $\mu_{l}+\sum_{i=1}^{m} \lambda_{i} \omega_{l}^{i}$ for every $l=1, \ldots, q$, as $\lambda_{i}>0$ for at least one index $i$. Consequently, $x \in \operatorname{rint} Q$.

Next result is a generalization of the well-known Weyl Theorem.

Theorem 4.4 (Extended Weyl Theorem) Let $U, V, D \subset \mathbb{R}^{n}$ finite sets such that $U \cup V \neq \emptyset$.

(i) The e-polytope $\operatorname{conv}(U \mid V)$ is a bounded e-polyhedron.

(ii) The Minkowski sum $\operatorname{conv}(U \mid V)+\operatorname{cone}(D)$ is an e-polyhedron.

Proof. Let $U=\left\{u_{1}, \ldots, u_{m}\right\}, V=\left\{v_{1}, \ldots, v_{q}\right\}$ and $D=\left\{d_{1}, \ldots, d_{r}\right\}$.

(i) Given $x \in \mathbb{R}^{n}, x \in \operatorname{conv}(U \mid V)$ if and only if the linear system with unknowns $\lambda_{i}(i=1, \ldots, m)$ and $\mu_{j}(j=1, \ldots, q)$

$$
\left\{x=\sum_{i=1}^{m} \lambda_{i} u_{i}+\sum_{j=1}^{q} \mu_{j} v_{j}, \sum_{i=1}^{m} \lambda_{i}+\sum_{j=1}^{q} \mu_{j}=1, \sum_{i=1}^{m} \lambda_{i}>0, \lambda_{i} \geq 0, \mu_{j} \geq 0\right\}
$$

is consistent. Hence, $\operatorname{conv}(U \mid V)$ is the set of solutions of the outcome system from removing the unknowns $\lambda_{i}$ and $\mu_{j}$ in the system with unknowns $\lambda_{i}, \mu_{j}$ and $x_{k}(k=$ $1, \ldots, n)$

$$
\left\{x_{k}=\sum_{i=1}^{m} \lambda_{i} u_{i k}+\sum_{j=1}^{q} \mu_{j} v_{j k}, \sum_{i=1}^{m} \lambda_{i}+\sum_{j=1}^{q} \mu_{j}=1, \sum_{i=1}^{m} \lambda_{i}>0, \lambda_{i} \geq 0, \mu_{j} \geq 0\right\} .
$$


By applying the Gauss-Fourier elimination method $m+q$ times, one has that $\operatorname{conv}(U \mid V)$ is an e-polyhedron. Furthermore, it is bounded as $\|x\| \leq \sum_{i=1}^{m}\left\|u_{i}\right\|+\sum_{j=1}^{q}\left\|v_{j}\right\|$ for all $x \in \operatorname{conv}(U \mid V)$.

(ii) By following the same reasoning as above, $\operatorname{conv}(U \mid V)+\operatorname{cone}(D)$ is an epolyhedron too, since $x \in \operatorname{conv}(U \mid V)+\operatorname{cone}(D)$ if and only if

$$
x=\sum_{i=1}^{m} \lambda_{i} u_{i}+\sum_{j=1}^{q} \mu_{j} v_{j}+\sum_{l=1}^{r} \alpha_{l} d_{l}
$$

for some $\lambda \in \mathbb{R}_{+}^{m}, \mu \in \mathbb{R}_{+}^{q}, \alpha \in \mathbb{R}_{+}^{r}, \sum_{i=1}^{m} \lambda_{i}+\sum_{j=1}^{q} \mu_{j}=1$ and $\sum_{i=1}^{m} \lambda_{i}>0$.

The first statement in the above theorem has already been proved in Lemma 4.2. However, the proof of Theorem 4.4 provides a method for obtaining an external representation of an e-polytope, and this method also applies for e-polyhedra.

Example 4.5 Consider the e-polytope $Q=\operatorname{conv}(U \mid V)$ in $\mathbb{R}^{2}$ described by the sets $U=\left\{(1,0),\left(\frac{3}{2}, 1\right)\right\}$ and $V=\{(0,0),(2,0),(1,2)\}$. Then, a given vector $\left(x_{1}, x_{2}\right) \in \mathbb{R}^{2}$ belongs to $Q$ if and only if the system

$$
\begin{array}{rllllll}
\lambda_{1}+\frac{3}{2} \lambda_{2} & & +2 \mu_{2} & +\mu_{3} & =x_{1} & \\
& \lambda_{2} & & +2 \mu_{3} & =x_{2} & \\
\lambda_{1}+\lambda_{2}+\mu_{1}+\mu_{2} & +\mu_{3} & =1 & \\
\lambda_{1}+\lambda_{2} & & & >0 & \\
& & & \lambda_{i} & \geq 0 & \forall i=1,2 . \\
& & \mu_{i} & \geq 0 & \forall i=1,2,3 .
\end{array}
$$

has a solution for $\lambda \in \mathbb{R}^{2}, \mu \in \mathbb{R}^{3}$. By applying the Gauss-Fourier method in order to eliminate the variables $\lambda$, $\mu$, one gets an external representation of $Q$,

$$
Q=\left\{\left(x_{1}, x_{2}\right) \in \mathbb{R}^{2}: 2 x_{1}+x_{2}-4 \leq 0,-x_{2} \leq 0,2 x_{1}-x_{2}-4<0,-2 x_{1}+x_{2}<0\right\} .
$$

Next theorem establishes an internal representation for e-polyhedra. It is the main result in this section and it generalizes the classical Motzkin theorem.

Theorem 4.6 (Decomposition of e-polyhedra) Let $P \subset \mathbb{R}^{n}$ be a nonempty epolyhedron. Then, $P$ is the Minkowski sum of an e-polytope and a polyhedral cone.

Proof. Let $P$ be the e-polyhedron given in Definition 3.1 and let $\mathcal{K}$ and $F_{n+2}$ be the polyhedral cones introduced in Definition 3.2.

$\mathcal{K}$ is a finitely generated cone, and so it is a polyhedral cone as a consequence of Lemma 2.1. Thus, $\mathcal{K}$ is the solution set of certain finite linear homogeneous system, say

$$
\mathcal{K}=\left\{(x, y, z) \in \mathbb{R}^{n} \times \mathbb{R} \times \mathbb{R}:\left\langle\left(\chi_{p}, \psi_{p}, \omega_{p}\right),(x, y, z)\right\rangle \leq 0, p=1, \ldots, s\right\} .
$$

This means that $\mathcal{K}=\left[\operatorname{cone}\left\{\left(\chi_{p}, \psi_{p}, \omega_{p}\right), p=1, \ldots, s\right\}\right]^{\circ}$, and so, by taking polars,

$$
\mathcal{K}^{\circ}=F_{n+2}=\operatorname{cone}\left\{\left(\chi_{p}, \psi_{p}, \omega_{p}\right), p=1, \ldots, s\right\} .
$$


We may assume without loss of generality that $F_{n+2}$ has just three kind of generators such that

$$
F_{n+2}=\text { cone }\left\{\left(u_{i},-1, \varepsilon_{i}\right), i=1, \ldots, m ;\left(v_{j},-1,0\right), j=1, \ldots, q ;\left(d_{l}, 0,0\right), l=1, \ldots, r\right\}
$$

with $-1 \leq \varepsilon_{i}<0$ for all $i=1, \ldots, m$ and $m+q+r=s$. Observe that there is at least one generator of the form $\left(u_{i},-1, \varepsilon_{i}\right)$, otherwise $F_{n+2} \subset\left\{(x, y, z) \in \mathbb{R}^{n+2}: z=0\right\}$ and so $P$ would be empty according to Lemma $3.5(i i)$, which contradicts the assumption.

Let $x \in P$. By Lemma $3.5(i)$, there exists $\varepsilon \in\left[-1,0\left[\operatorname{such}\right.\right.$ that $(x,-1, \varepsilon) \in F_{n+2}$. Therefore, there exist $\lambda \in \mathbb{R}_{+}^{m}, \mu \in \mathbb{R}_{+}^{q}$ and $\alpha \in \mathbb{R}_{+}^{r}$ such that

$$
(x,-1, \varepsilon)=\sum_{i=1}^{m} \lambda_{i}\left(u_{i},-1, \varepsilon_{i}\right)+\sum_{j=1}^{q} \mu_{j}\left(v_{j},-1,0\right)+\sum_{l=1}^{r} \alpha_{l}\left(d_{l}, 0,0\right) .
$$

Thus, one has

$$
x=\sum_{i=1}^{m} \lambda_{i} u_{i}+\sum_{j=1}^{q} \mu_{j} v_{j}+\sum_{l=1}^{r} \alpha_{l} d_{l}
$$

with $\sum_{i=1}^{m} \lambda_{i}+\sum_{j=1}^{q} \mu_{j}=1$ and $\sum_{i=1}^{m} \lambda_{i} \geq \sum_{i=1}^{m}-\varepsilon_{i} \lambda_{i}=-\varepsilon>0$. Hence, by letting $U:=\left\{u_{1}, \ldots, u_{m}\right\}, V:=\left\{v_{1}, \ldots, v_{q}\right\}$ and $D:=\left\{d_{1}, \ldots, d_{r}\right\}$, one has

$$
P=\operatorname{conv}(U \mid V)+\operatorname{cone}(D)
$$

and the conclusion follows.

The above decomposition theorem holds true for any e-polyhedron, and so, for any (closed convex) polyhedron in particular. However, the classical decomposition of polyhedra is given by means of a polytope and a polyhedral cone. Hence, we should obtain such a decomposition as a consequence of Theorem 4.6.

Corollary 4.7 (Motzkin Theorem) Let $P \subset \mathbb{R}^{n}$ be a nonempty polyhedron. Then, $P$ is the sum of a polytope and a polyhedral cone.

Proof. By applying Theorem 4.6, there exist finite sets $U, V, D \subset \mathbb{R}^{n}$ such that $P=\operatorname{conv}(U \mid V)+\operatorname{cone}(D)$. As $P$ is closed, $P=\operatorname{cl} P$. On the other hand,

$$
\mathrm{cl}(\operatorname{conv}(U \mid V)+\operatorname{cone}(D))=\operatorname{cl} \operatorname{conv}(U \mid V)+\operatorname{cone}(D)=\operatorname{conv}(U \cup V)+\operatorname{cone}(D) .
$$

Hence, $P=\operatorname{conv}(U \cup V)+\operatorname{cone}(D)$ and the conclusion follows.

The internal representation for e-polyhedra obtained in Theorem 4.6 coincides with the one given in $[1$, Theorem 4.4]. Our proof, instead, does not use the standard version of Motzkin theorem but a generalization of the technique employed by Motzkin. On the other hand, the given proof of Theorem 4.6 provides a method for obtaining an internal representation of an e-polyhedron. Let $P$ be the e-polyhedron introduced in Definition 3.1 and $\mathcal{K}$ its associated extended characteristic cone. By applying the Gauss-Fourier 
method we shall obtain an external representation of $\mathcal{K}$. It can be assumed without loss of generality that such a representation has the form

$$
\mathcal{K}=\left\{\begin{aligned}
\left\langle\left(u_{i},-1, \varepsilon_{i}\right),(x, y, z)\right\rangle & \leq 0, i=1, \ldots, m \\
(x, y, z) \in \mathbb{R}^{n+2}: & \left\langle\left(v_{j},-1,0\right),(x, y, z)\right\rangle \\
\left\langle\left(d_{l}, 0,0\right),(x, y, z)\right\rangle & \leq 0, j=1, \ldots, l=1, \ldots, r
\end{aligned}\right\}
$$

where $-1 \leq \varepsilon_{i}<0$ for all $i=1, \ldots, m$. Then, one has

$$
P=\operatorname{conv}\left(\left\{u_{1}, \ldots, u_{m}\right\} \mid\left\{v_{1}, \ldots, v_{q}\right\}\right)+\operatorname{cone}\left(d_{1}, \ldots, d_{r}\right) .
$$

Next example illustrates how this method works.

Example 4.8 Consider the e-polyhedron $P$ in $\mathbb{R}^{2}$ (see $[5$, Example 2.1]) defined by

$$
P:=\left\{\left(x_{1}, x_{2}\right) \in \mathbb{R}^{2}:-x_{1}-3 x_{2} \leq-2,-x_{1} \leq 0,-3 x_{1}-x_{2}<-2,-x_{2}<0\right\} .
$$

The extended characteristic cone $\mathcal{K}$ associated to $P$ is the cone finitely generated by $(-1,-3,-2,0),(-1,0,0,0),(-3,-1,-2,-1),(0,-1,0,-1),(0,0,1,-1)$ and $(0,0,0,1)$. Hence, a given vector $\left(x_{1}, x_{2}, y, z\right) \in \mathbb{R}^{4}$ belongs to $\mathcal{K}$ if and only if the linear system

$$
\begin{aligned}
& \begin{array}{llll}
-\lambda_{1} & -\lambda_{2} & -3 \lambda_{3} & =
\end{array} \\
& \begin{array}{llll}
-3 \lambda_{1} & -\lambda_{3} & -\lambda_{4} & =x_{2}
\end{array} \\
& \begin{array}{llll}
-2 \lambda_{1} & -2 \lambda_{3} & +\lambda_{5} & =y
\end{array}
\end{aligned}
$$

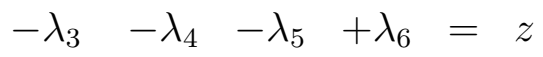

$$
\begin{aligned}
& \lambda_{i} \geq 0 \quad \forall i=1, \ldots, 6 .
\end{aligned}
$$

has a solution for $\lambda \in \mathbb{R}^{6}$. By applying the Gauss-Fourier method in order to eliminate the variables $\lambda$, one gets the following external representation of $\mathcal{K}$ :

$$
\begin{array}{ccc}
\frac{2}{3} x_{1}+\frac{4}{9} x_{2}-y-\frac{4}{9} z \leq 0, & 3 x_{2}-y-z \leq 0, & \frac{2}{3} x_{1}+\frac{2}{3} x_{2}-y-\frac{2}{3} z \leq 0 \\
\frac{2}{3} x_{1}+x_{2}-y-z \leq 0, & \frac{8}{3} x_{2}-y-\frac{2}{3} z \leq 0, & \frac{1}{2} x_{1}+\frac{1}{2} x_{2}-y \leq 0 \\
2 x_{1}-y \leq 0, & 2 x_{2}-y \leq 0, & \frac{4}{9} x_{1}+\frac{2}{3} x_{2}-y \leq 0 \\
x_{1} \leq 0, & x_{2} \leq 0 . &
\end{array}
$$

Thus, according to the above method, an internal representation of $P$ is given by $\operatorname{conv}(U \mid V)+\operatorname{cone}(D)$ where

$$
\begin{aligned}
U & =\left\{\left(\frac{2}{3}, \frac{4}{9}\right),(0,3),\left(\frac{2}{3}, \frac{2}{3}\right),\left(\frac{2}{3}, 1\right),\left(0, \frac{8}{3}\right)\right\} \\
V & =\left\{\left(\frac{1}{2}, \frac{1}{2}\right),(2,0),(0,2),\left(\frac{4}{9}, \frac{2}{3}\right)\right\} \\
D & =\{(1,0),(0,1)\}
\end{aligned}
$$

Next result is a straightforward consequence of Theorems 4.4 and 4.6.

Corollary 4.9 (Characterization of e-polyhedra) Let $P \subset \mathbb{R}^{n}$. One has:

(i) $P$ is an e-polyhedron if and only if it is the Minkowski sum of an e-polytope and a polyhedral cone. 
(ii) $P$ is an e-polytope if and only if it is a bounded e-polyhedron.

Now, by employing the notion of minimal face, we can prove a refinement of Theorem 4.6.

Theorem 4.10 (Minimal decomposition of e-polyhedra) Let $P \subset \mathbb{R}^{n}$ be a nonempty e-polyhedron. Then,

$$
P=\operatorname{conv}(U \mid V)+\operatorname{rec}(P)
$$

where $U:=\left\{u_{1}, \ldots, u_{m}\right\}, u_{i} \in C_{i}$ with $C_{i}$ a minimal face of $P$, for every $i=1, \ldots, m$, and $V:=\left\{v_{1}, \ldots, v_{q}\right\}, v_{j} \in D_{j}$ with $D_{j}$ a minimal face of $\bar{P}$ not intersecting $P$, for every $j=1, \ldots, q$.

Proof. Assume that $P$ is the solution set of the consistent system $\sigma$ in (1) and recall that $\mathrm{cl} P=\bar{P}:=\left\{x \in \mathbb{R}^{n}:\left\langle a_{t}, x\right\rangle \leq b_{t}, t \in W \cup S\right\}$. We shall denote by $G$ the term in the right-hand side of $(10)$.

Let $\bar{x} \in \operatorname{conv}(U \mid V)$. Then, there exist $\lambda \in \mathbb{R}_{+}^{m}$ and $\mu \in \mathbb{R}_{+}^{q}$ with $\sum_{i=1}^{m} \lambda_{i}+$ $\sum_{j=1}^{q} \mu_{j}=1$ and $\sum_{i=1}^{m} \lambda_{i}>0$ such that

$$
\bar{x}=\sum_{i=1}^{m} \lambda_{i} u_{i}+\sum_{j=1}^{q} \mu_{j} v_{j} .
$$

As $u_{i} \in P$ for any $i=1, \ldots, m, v_{j} \in \bar{P}$ for any $j=1, \ldots, q$, and $\sum_{i=1}^{m} \lambda_{i}>0$, it is easy to check that $\left\langle a_{t}, \bar{x}\right\rangle \leq b_{t}$ for all $t \in W$ and $\left\langle a_{t}, \bar{x}\right\rangle<b_{t}$ for all $t \in S$, which means $\bar{x} \in P$. Thus, $\operatorname{conv}(U \mid V) \subset P$ and so, $G:=\operatorname{conv}(U \mid V)+\operatorname{rec}(P) \subset P$.

To obtain the identity in (10), we assume that there exists $\bar{x} \in P \backslash G$ and we shall get a contradiction. For that purpose we observe that, in virtue of Theorem 4.4, $G$ is an e-polyhedron and so, it is the solution set of certain finite linear system, say

$$
G=\left\{x \in \mathbb{R}^{n}:\left\langle c_{t}, x\right\rangle \leq e_{t}, t \in \bar{W} ;\left\langle c_{t}, x\right\rangle<e_{t}, t \in \bar{S}\right\}
$$

for certain $\left(c_{t}, e_{t}\right) \in \mathbb{R}^{n} \times \mathbb{R}$ for all $t \in \bar{W} \cup \bar{S}$ with $|\bar{W} \cup \bar{S}|<\infty$, and as $G$ is nonempty, one also has $\operatorname{cl} G=\bar{G}:=\left\{x \in \mathbb{R}^{n}:\left\langle c_{t}, x\right\rangle \leq e_{t}, t \in \bar{W} \cup \bar{S}\right\}$. Recall that $U \subset G$ and $U \cup V \subset \operatorname{cl} G=\operatorname{conv}(U \cup V)+\operatorname{rec}(P)$. Hence, if $\bar{x} \notin G$, either $\left\langle c_{t_{1}}, \bar{x}\right\rangle>e_{t_{1}}$ for some $t_{1} \in \bar{W} \cup \bar{S}$, or $\left\langle c_{t_{2}}, \bar{x}\right\rangle=e_{t_{2}}$ for some $t_{2} \in \bar{S}$. Next we study these two cases separately.

Case 1: There exists $t_{1} \in \bar{W} \cup \bar{S}$ such that $\left\langle c_{t_{1}}, \bar{x}\right\rangle>e_{t_{1}}$. Then, as $\bar{x} \in P$, one has

$$
\alpha_{1}:=\max _{x \in \bar{G}}\left\langle c_{t_{1}}, x\right\rangle=\sup _{x \in G}\left\langle c_{t_{1}}, x\right\rangle \leq e_{t_{1}}<\left\langle c_{t_{1}}, \bar{x}\right\rangle \leq \sup _{x \in P}\left\langle c_{t_{1}}, x\right\rangle=\max _{x \in \bar{P}}\left\langle c_{t_{1}}, x\right\rangle=: \beta_{1} .
$$

We first observe that $\beta_{1} \in \mathbb{R}$, otherwise, if $\beta_{1}=+\infty$ there would be $d \in \operatorname{rec}(\bar{P})$ such that $\left\langle c_{t_{1}}, d\right\rangle>0$ which entails $\alpha_{1}=+\infty$, since $\operatorname{rec}(\bar{P})=\operatorname{rec}(\bar{Q})$, and thus a contradiction. Thence, $\bar{F}:=\left\{x \in \bar{P}:\left\langle c_{t_{1}}, x\right\rangle=\beta_{1}\right\}$ is a nonempty face of $\bar{P}$, and let $F:=\bar{F} \cap P$.

If $F \neq \emptyset$, then $F=\left\{x \in P:\left\langle c_{t_{1}}, x\right\rangle=\beta_{1}\right\}$ is a nonempty face of $P$. Let $u_{i} \in C_{i}$ with $C_{i}$ being a minimal face of $F$ and so, a minimal face of $P$. Then, $\left\langle c_{t_{1}}, u_{i}\right\rangle=\beta_{1}>e_{t_{1}}$ which contradicts the fact that $u_{i} \in G$. 
Otherwise, if $F=\emptyset$, let $v_{j} \in D_{j}$ with $D_{j}$ being a minimal face of $\bar{F}$ and so, a minimal face of $\bar{P}$, which clearly does not intersect $P$. Then, $\left\langle c_{t_{1}}, v_{j}\right\rangle=\beta_{1}>e_{t_{1}}$ which contradicts the fact that $v_{j} \in \operatorname{cl} G$.

Case 2: There exists $t_{2} \in \bar{S}$ such that $\left\langle c_{t_{2}}, \bar{x}\right\rangle=e_{t_{2}}$. Then, as $\bar{x} \in P$, one has

$$
\max _{x \in \bar{G}}\left\langle c_{t_{2}}, x\right\rangle=\sup _{x \in G}\left\langle c_{t_{2}}, x\right\rangle \leq e_{t_{2}}=\left\langle c_{t_{2}}, \bar{x}\right\rangle \leq \sup _{x \in P}\left\langle c_{t_{2}}, x\right\rangle=\max _{x \in \bar{P}}\left\langle c_{t_{2}}, x\right\rangle=: \beta_{2} .
$$

Reasoning as in the Case 1 , one has $\beta_{2} \in \mathbb{R}$ and so $\bar{F}:=\left\{x \in \bar{P}:\left\langle c_{t_{2}}, x\right\rangle=\beta_{2}\right\}$ is a nonempty face of $\bar{P}$. Let $F:=\bar{F} \cap P$.

If $F \neq \emptyset$, then $F=\left\{x \in P:\left\langle c_{t_{2}}, x\right\rangle=\beta_{2}\right\}$ is a nonempty face of $P$. Let $u_{i} \in C_{i}$ with $C_{i}$ being a minimal face of $F$ and so, a minimal face of $P$. Then, $\left\langle c_{t_{2}}, u_{i}\right\rangle=\beta_{2} \geq e_{t_{2}}$ which contradicts the fact that $u_{i} \in G$.

Otherwise, if $F=\emptyset$, let $v_{j} \in D_{j}$ with $D_{j}$ being a minimal face of $\bar{F}$ and so, a minimal face of $\bar{P}$, which clearly does not intersect $P$. Then, $\left\langle c_{t_{2}}, v_{j}\right\rangle=\beta_{2} \geq e_{t_{2}}$. Observe that if $\beta_{2}=e_{t_{2}}$ then $\bar{x} \in \bar{F} \cap P$ and the assumption $F=\emptyset$ would fail. Hence, one has $\left\langle c_{t_{2}}, v_{j}\right\rangle=\beta_{2}>e_{t_{2}}$ which contradicts the fact that $v_{j} \in \mathrm{cl} G$.

Example 4.11 Let consider again the e-polyhedron $P$ defined in Example 4.8. The minimal faces of $P$ are $C_{1}=\left\{\left(x_{1}, x_{2}\right) \in \mathbb{R}^{2}: 3 x_{2}=-x_{1}+2,1<2 x_{1}<4\right\}$ and $C_{2}=\left\{\left(x_{1}, x_{2}\right) \in \mathbb{R}^{2}: x_{1}=0, x_{2} \geq 2\right\}$, whereas the minimal faces of $\bar{P}$ not intersecting $P$ are $D_{1}=\left\{\left(\frac{1}{2}, \frac{1}{2}\right)\right\}, D_{2}=\{(2,0)\}$ and $D_{3}=\{(0,2)\}$. The extreme rays of $P$ are $d_{1}=(1,0)$ and $d_{2}=(0,1)$, and so $\operatorname{rec}(P)=\operatorname{cone}\left(d_{1}, d_{2}\right)$. Then, by choosing any $u_{1} \in C_{1}$ and $u_{2} \in C_{2}$, Theorem 4.10 guarantees that an internal representation of $P$ is

$$
P=\operatorname{conv}\left(\left\{u_{1}, u_{2}\right\} \mid\left\{\left(\frac{1}{2}, \frac{1}{2}\right),(2,0),(0,2)\right\}\right)+\operatorname{cone}\left(d_{1}, d_{2}\right) .
$$

In particular, as $\left(\frac{2}{3}, \frac{4}{9}\right) \in C_{1}$ and $(0,3) \in C_{2}$, then (10) holds with

$$
\begin{aligned}
U & =\left\{\left(\frac{2}{3}, \frac{4}{9}\right),(0,3)\right\}, \\
V & =\left\{\left(\frac{1}{2}, \frac{1}{2}\right),(2,0),(0,2)\right\} .
\end{aligned}
$$

Thus, we observe that the representation given in Example 4.8 was not minimal in the sense that the sets of generators there can be simplified as shown.

As a straightforward consequence of Theorem 4.10 we obtain the following internal representations for e-polyhedra which are either closed or relatively open.

Corollary 4.12 Let $P \subset \mathbb{R}^{n}$ be a nonempty e-polyhedron.

(i) If $P$ is closed, then $P=\operatorname{conv}\left(u_{1}, \ldots, u_{m}\right)+\operatorname{rec}(P)$ with $u_{i} \in C_{i}$ and $C_{i}$ a minimal face of $P$, for every $i=1, \ldots, m$.

(ii) If $P$ is relatively open, then $P=\operatorname{conv}\left(u \mid v_{1}, \ldots, v_{q}\right)+\operatorname{rec}(P)$ with $u \in P, v_{j} \in D_{j}$ and $D_{j}$ a minimal face of $\bar{P}$ not intersecting $P$, for every $j=1, \ldots, q$.

Thus, if a (closed convex) polyhedron contains no line, it is the sum of its recession cone and the convex hull of its extreme points. The above internal representation for relatively open polyhedral sets differs from the one given in 5 , Lemma 2.3]. Just as an illustration, the e-polyhedron $P=\left\{x \in \mathbb{R}^{2}: x_{1}>1, x_{2}>1\right\}$ can be represented as stated in Corollary 4.12 by $\operatorname{conv}(u \mid v)+\mathbb{R}_{+}^{2}$ where $v=(1,1)$ and $u$ is any point in $P$. 


\section{Conclusions}

The current study is concerned with the study of solution sets of finite linear systems possibly containing strict inequalities, the so-called e-polyhedra. Our starting point is Motzkin theorem, which states that every polyhedron can be represented as the Minkowski sum of a polytope and a polyhedral cone. The technique used for obtaining this representation consists in building a polyhedral cone into a one higher dimension space. We adapt this technique in order to obtain the representation of an e-polyhedron as the Minkowki sum of an e-polytope and a polyhedral cone. For that purpose, we build a polyhedral cone into a two higher dimension space, referred to as extended characteristic cone. Intuitively, if the given e-polyhedron is the solution set of certain finite linear system in $\mathbb{R}^{n}$, then the $n+1$ first components of that cone are associated to the data of the system whereas the last component is associated to the kind of inequality (either weak or strict). As every (closed convex) polyhedron is an e-polyhedron, we thus obtain a generalization of Motzkin theorem. In addition to that, we provide consistency and Farkas-type results in terms of this new dual cone, and a generalization of Weyl theorem. In this way, our results achieve the claims in $\lfloor 1\rfloor$ and possibly let study, in a further research, a new and more direct encoding of e-polyhedra as requested in $\lfloor 2\rfloor$.

\section{Acknowledgements}

This research was partially supported by MINECO of Spain and ERDF of EU, Grant MTM2014-59179-C2-1-P.

\section{References}

[1] Bagnara R, Hill PM, Zaffanella E (2005) Not necessarily closed convex polyhedra and the double description method. Formal Aspects of Computing 17:222-257.

[2] Bagnara R, Hill PM, Zaffanella E (2009) Applications of polyhedral computations to the analysis and verification of hardware and software systems. Theoretical Computer Science 410:4672-4691.

[3] Bagnara R, Ricci E, Zaffanella E, Hill PM (2002) Possibly not closed convex polyhedra and the Parma Polyhedra Library. Proceedings 9th International Static Analysis Symposium (Madrid) 213-229.

[4] Fang YP, Huang NJ, Yang XQ (2012a) Local smooth representations of parametric semiclosed polyhedra with applications to sensitivity in piecewise linear programs. J. Optim. Theory Appl. 155:810-839.

[5] Fang YP, Meng K, Yang XQ (2012b) Piecewise linear multicriteria programs: The continuous case and its discontinuous generalization. Operations Research 60(2):398-409. 
[6] Farkas J (1902) Theorie der einfachen Ungleichungen. J. Reine Angew. Math. 124:1-27.

[7] Fenchel W (1952) A remark on convex sets and polarity. Communications du Séminaire Mathématique de l'Université de Lund, Supplement:82-89.

[8] Goberna MA, Jeyakumar V, Dihn N (2006) Dual characterizations of set containments with strict convex inequalities. J. Global Optim. 34:33-54.

[9] Goberna MA, Jornet V, Puente R (2004) Optimización Lineal: Teoría, Métodos y Modelos (McGraw-Hill, Madrid).

[10] Goberna MA, Jornet V, Rodríguez MML (2003) On linear systems containing strict inequalities. Linear Algebra and its Applications 360:151-171.

[11] Goberna MA, López MA (1998) Linear semi-Infinite optimization (John Wiley \& Sons, Chichester).

[12] Goberna MA, Rodríguez MML (2006) Analyzing linear systems containing strict inequalities via evenly convex hulls. European J. Oper. Res. 169:1079-1095.

[13] Kannan R (1992) Lattice translates of a polytope and the Frobenius problem. Combinatorica 12:161-177.

[14] Klee V (1959) Some characterizations of convex polyhedra. Acta Math. 102:79-107.

[15] Klee V (1966) Convex polytopes and linear programming. Proceedings IBM Scientific Computing Symposium on Combinatorial Problems (New York) 123-158.

[16] Kuhn HW (1956) Solvability and consistency for linear equations and inequalities. Amer. Math. Monthly 63:217-232.

[17] Kuhn HW, Tucker AW, eds. (1956) Linear inequalities and related systems. Annals of Mathematics Studies 38 (Princeton, NJ).

[18] Minkowski H (1896) Geometrie der Zahlen (Erste Lieferung) (Teubner, Leipzig).

[19] Motzkin TS (1936) Beiträge zur theorie der linearen ungleichungen (Azriel, Jerusalem). Transl. in: Cantor D, Gordon B, Rothschild B, eds. (1983) Theodore S. Motzkin: Selected papers (Birkhäuser, Boston), 1-80.

[20] Murty KG (2010) Optimization for Decision Making. Linear and Quadratic Models. International Series in Operations Research \& Management Science (Springer New York).

[21] Rockafellar RT (1970) Convex Analysis (Princeton Univ. Press, Princeton, NJ).

[22] Schrijver A (1986) Theory of linear and integer programming. Wiley-Interscience Series in Discrete Mathematics (John Wiley \& Sons, Chichester). 
[23] Schrijver A (2004) Combinatorial Optimization. Polyhedra and Efficiency (Springer-Verlag Berlin, Heidelberg).

[24] Stoer J, Witzgall C (1970) Convexity and optimization in finite dimensions I (Springer-Verlag Berlin, Heidelberg).

[25] Szilágyi P (1999) Nonhomogeneous linear theorems of the alternative. Pure Mathematics and Applications 10:141-159.

[26] Walkup DW, Wets RJ (1969) A Lipschitzian characterization of convex polyhedra. Proc. Amer. Math. Soc. 23:167-173.

[27] Weyl H (1935) Elementare theorie der konvexen polyeder. Comment. Math. Helv. $7: 290-306$.

[28] Yang XQ, Yen ND (2010) Structure and weak sharp minimum of the Pareto solution set for piecewise linear multiobjective optimization. J. Optim. Theory Appl. $147: 113-124$.

[29] Zheng XY (2009) Pareto solutions of polyhedral-valued vector optimization problems in Banach spaces. Set-Valued Var. Anal 17:389-408.

[30] Zhu YJ (1966) Generalizations of some fundamental theorems on linear inequalities. Acta Math. Sinica 16:25-39. 